\title{
Supersymmetric Quantum Mechanics and Super-Lichnerowicz Algebras
}

\author{
K. Hallowell and A. Waldron \\ Department of Mathematics, University of California, Davis CA 95616, USA \\ hallowell, wally@math.ucdavis.edu \\ Dedicated to the memory of Tom Branson
}

\begin{abstract}
We present supersymmetric, curved space, quantum mechanical models based on deformations of a parabolic subalgebra of $\operatorname{ssp}(2 p+2 \mid Q)$. The dynamics are governed by a spinning particle action whose internal coordinates are Lorentz vectors labeled by the fundamental representation of $\operatorname{osp}(2 p \mid Q)$. The states of the theory are tensors or spinor-tensors on the curved background while conserved charges correspond to the various differential geometry operators acting on these. The Hamiltonian generalizes Lichnerowicz's wave/Laplace operator. It is central, and the models are supersymmetric whenever the background is a symmetric space, although there is an $\operatorname{ssp}(2 p \mid Q)$ superalgebra for any curved background. The lowest purely bosonic example $(2 p, Q)=(2,0)$ corresponds to a deformed Jacobi group and describes Lichnerowicz's original algebra of constant curvature, differential geometric operators acting on symmetric tensors. The case $(2 p, Q)=(0,1)$ is simply the $\mathcal{N}=1$ superparticle whose supercharge amounts to the Dirac operator acting on spinors. The $(2 p, Q)=(0,2)$ model is the $\mathcal{N}=2$ supersymmetric quantum mechanics corresponding to differential forms. (This latter pair of models are supersymmetric on any Riemannian background.) When $Q$ is odd, the models apply to spinor-tensors. The $(2 p, Q)=(2,1)$ model is distinguished by admitting a central Lichnerowicz-Dirac operator when the background is constant curvature. The new supersymmetric models are novel in that the Hamiltonian is not just a square of super charges, but rather a sum of commutators of supercharges and commutators of bosonic charges. These models and superalgebras are a very useful tool for any study involving high rank tensors and spinors on manifolds.
\end{abstract}




\section{Contents}

1 Introduction $\quad 2$

2 The Model 6

2.1 Orthosymplectic Superalgebras ... . . . . . . . . . 6

2.2 Orthosymplectic Spinning Particle . . . . . . . . . . 7

3 Quantization 9

3.1 Canonical Analysis . . . . . . . . . . . . . . . . . . 9 9

3.2 States . . . . . . . . . . . . . . . . . 10

3.3 Conserved Charges . . . . . . . . . . . . . . . 12

4 Geometry $\quad \mathbf{1 5}$

4.1 Dirac Operators and $\mathcal{N}=1$ Supersymmetry . . . . . . . . . 15

4.2 Differential Forms and $\mathcal{N}=2$ Supersymmetry . . . . . . . 15

4.3 Symmetric Algebras and Quantum Mechanics . . . . . . . 16

4.4 Lichnerowicz Dirac Operator . . . . . . . . . . . . . . . . . 20

4.5 Multisymmetric Tensors . . . . . . . . . . . . . . 22

4.6 Multiforms . . . . . . . . . . . . . . . . . . 25

4.7 Super-Lichnerowicz Algebras . . . . . . . . . . . . . . . . 27

4.8 Parabolic Orthosymplectic Algebra . . . . . . . . . . . . 30

5 Conclusions $\quad 32$ 


\section{Introduction}

Since the early 1980's it has been clear that supersymmetric quantum mechanical models are deeply related to geometry. In particular, AlvarezGaumé and Witten computed gravitational anomalies and Pontryagin classes by identifying the Dirac operator on a Riemannian manifold with the supercharge of an $\mathcal{N}=1$ supersymmetric quantum mechanics [1]. In an application to Morse theory, Witten realized that the supercharges of $\mathcal{N}=2 \mathrm{su}-$ persymmetric quantum mechanics corresponded to the exterior derivative $\mathbf{d}$ and codifferential $\boldsymbol{\delta}[2]$. In these models the Hamiltonian corresponds to the Laplace operator, and states are spinors or differential forms, respectively. Increasing the number of supersymmetries to $\mathcal{N}=4$ requires that the background manifold be Kähler [3] and the supersymmetry charges are now the Dolbeault operators. In particular the $s l(2, \mathbb{R})$ Hodge-Lefschetz algebra of the de Rham cohomology of a Kähler manifold amounts to the algebra of conserved charges of the $\mathcal{N}=4$ supersymmetric quantum mechanics $[4,5]$.

If instead of studying differential forms on a manifold $M$, but rather symmetric tensors, there is also an extremely useful algebra of the gradient and divergence operations. In particular, Lichnerowicz observed that for constant curvature backgrounds one could introduce a wave operator that generalized the Laplacian and (in some sense) commuted with the action of the divergence and gradient operators on symmetric tensors [6]. (His original motivation was a computation of the spin 2 massive propagator on these spaces.) In a recent study of higher spins in constant curvature manifolds we found the algebra of the following operators on symmetric tensors ${ }^{1}$ [8]:

\begin{tabular}{c|c|c}
\hline Index & $\mathbf{N}$ & Counts indices. \\
Trace & $\mathbf{t r}$ & Traces a pair of indices. \\
Metric & $\mathbf{g}$ & Multiplies by the metric and symmetrizes. \\
Divergence & $\mathbf{d i v}$ & The covariant divergence. \\
Gradient & grad & Symmetrized covariant derivative. \\
\hline
\end{tabular}

The result was a deformation of the Jacobi group (a central extension of $\left.s l(2, \mathbb{R}) \ltimes \mathbb{R}^{2}\right)$ and in this paper we extend it to an arbitrary symmetric

\footnotetext{
${ }^{1}$ The flat version of this algebra has been studied in the context of conformally flat manifolds in [7].
} 
space. In particular, unlike differential forms for which the anticommutator of the exterior derivative and codifferential (morally - gradient and divergence) yield the form Laplace operator

$$
\{\mathbf{d}, \boldsymbol{\delta}\}=\square
$$

for symmetric tensors, it is the commutator that does so

$$
[\operatorname{div}, \operatorname{grad}]=\Delta-\mathbf{g} \operatorname{tr}+\mathbf{N}\left(\mathbf{N}+\operatorname{dim}_{M}-2\right)
$$

Here $\mathbf{g} \mathbf{t r}-\mathbf{N}\left(\mathbf{N}+\operatorname{dim}_{M}-2\right)$ is the Casimir of the $s l(2, \mathbb{R})$ algebra generated by $(\mathbf{g}, \mathbf{N}, \mathbf{t r})$ and the central Lichnerowicz wave operator equals

$$
\square=\Delta+\mathbf{g} \operatorname{tr}-\mathbf{N}\left(\mathbf{N}+\operatorname{dim}_{M}-2\right) .
$$

The natural question, posed and answered here, is whether there exists a Lichnerowicz/Lefschetz type algebra applicable to both differential forms and symmetric tensors, or indeed, for the most general tensors and spinor-tensors on a Riemannian manifold. We answer this question in the affirmative and find that there exists a central Lichnerowicz wave operator $\square$ whenever the manifold $M$ is a locally symmetric space, namely its curvature is covariantly constant

$$
D_{\kappa} R_{\mu \nu \rho \sigma}=0 \text {. }
$$

The operator $\square$ also exists for general Riemannian manifolds and always commutes with an $\operatorname{ssp}(2 p \mid Q)$ subalgebra of operators generalizing $\{\mathbf{g}, \mathbf{N}, \mathbf{t r}\}$.

We construct these "super-Lichnerowicz" algebras from the conserved charges of a supersymmetric quantum mechanical model whose states are tensors and tensor-spinors on $M$. The model itself is a simple generalization of the $\mathcal{N}=2$ supersymmetric quantum mechanics. The action is just

$$
S=\frac{1}{2} \int d t\left\{\dot{x}^{\mu} g_{\mu \nu} \dot{x}^{\nu}-i X^{\mu} \cdot \frac{D X_{\mu}}{d t}+\frac{1}{4} R_{\mu \nu \rho \sigma} X^{\mu} \cdot X^{\nu} X^{\rho} \cdot X^{\sigma}\right\}
$$

or in other words, a generalized spinning particle model $[9,10,11]$. The fields $x^{\mu}(t)$ are the worldline imbedding coordinates, while $X^{\mu}(t)$ are a collection of both bose and fermi fields transforming under the fundamental representation of the superalgebra $\operatorname{osp}(2 p \mid Q)$ for which the dot "." denotes the invariant inner product. Supersymmetry requires the background to be a locally symmetric space. Gauged models of this type where $p=0$ and the 
fields $X^{\mu}$ are purely fermionic are known to describe massless higher spin particles $^{2}[12]$. (When $(2 p, Q)=(2,0)$, the fields $X^{\mu}$ carry an $s p(2)$ index corresponding to the spinor and conformal vector oscillators first introduced by Labastida and Vasiliev to describe higher spin fields $[14,15,16]$.)

The conserved charges of the model correspond to both commuting and anti-commuting generalizations of the gradient and divergence (alias exterior derivative and codifferential) operators, along with a Lichnerowicz wave operator (the Hamilitonian), and operators that trace over indices, count indices of a given type (form or totally symmetric), multiply by metric tensors. and change indices from one type to another. Taking the parameters $(2 p, Q)$ completely general lets us describe arbitrary tensors and spinor-tensors. The interpretations for some specific values are listed below

${ }_{(0,1)} \mathcal{N}=1$ supersymmetric quantum mechanics - describes the Dirac operator acting on spinors.

(0,2) $\mathcal{N}=2$ supersymmetric quantum mechanics - describes the exterior derivative acting on differential forms.

$(2,0)$ This model is purely bosonic and yields Lichnerowicz's original construction - states are symmetric tensors. The conserved charge algebra is the "symmetric algebra" of [8].

(2,1) A model of totally symmetric spinor-tensors (i.e., spinors with an arbitrary number of totally symmetric vector indices), which means there are charges corresponding to gamma-traces (contracting vector and spinor indices using Dirac matrices). This algebra was first encountered in a study of partially massless fermionic fields in [17] and sytematized in [8]. (It has also appeared in the computation of massive and partially massless fermionic actions [18].) The theory is distinguished by possessing a "Lichnerowicz-Dirac" operator - a modification of the Dirac operator that commutes with all other charges when the background is constant curvature (but not in general symmetric spaces).

$(2 \mathrm{p}, 0)$ A purely bosonic model, but states are now "multi-symmetric tensors". There are $p$ different gradient and divergence operators along with operators that remove or add pairs of indices or change index types.

\footnotetext{
${ }^{2}$ Recently, the one-loop quantization of these models has also been studied [13].
} 
(0,2q) States are multi-forms (indices are grouped into antisymmetric subsets, e.g., the Riemann tensor is a "bi-form"). There are now $q$ distinct exterior derivatives and codifferentials. Algebras of this type have been studied by Hull and Medeiros in the context of "exotic" higher spin gauge theories [19] and a mathematical analysis was given in [20, 21].

For $(2 p, Q)=(0,1)$ or $(0,2)$, namely the $\mathcal{N}=1,2$ supersymmetric models, the algebra of conserved charges is a finite dimensional superalgebra for any background Riemannian manifold. The same is true whenever the target manifold $M$ is flat but $(2 p, Q)$ are arbitrary. In general backgrounds, we obtain a deformation of the finite dimensional Lie superalgebra appearing in the flat case. The simplest example is the $(2,0)$ model in a constant curvature background subject to the algebra (2). The Lichnerowicz wave operator $\square$ is central but the $\operatorname{sl}(2, \mathbb{R})$ Casimir does not commute with the doublet (grad, div). Instead, further commutators yield an infinite series of higher operators (involving increasing powers of the "oscillators" $X^{\mu}$ ). In this case there exists a reformulation of the resulting infinite dimensional Lie algebra as a rather simple associative algebra, obtained by including a certain square root of the Casimir operator [8]. This is not a direction we pursue in this paper, but there is a simple characterization of the Lie superalgebra being deformed as a parabolic subalgebra $\mathfrak{p}$ of $\operatorname{osp}(2 p+2 \mid Q)$. This parabolic superalgebra can be viewed as a (supersymmetric) generalization of the Jacobi group $G^{J}=\left(S l(2, \mathbb{R}) \ltimes \mathbb{R}^{2}\right) \times R^{*}$.

Our results are organized as follows: In section 2 , we briefly review $\operatorname{osp}(2 p \mid Q)$ superalgebras and write down our supersymmetric quantum mechanical model. Then we quantize it and determine its Hilbert space, conserved charges, and their algebra in section 3. Section 4 is concerned with the application of the model to geometry. There we discuss each of the special examples itemized above, followed by the most general case and also the deformed parabolic superalgebra of differential geometric operators on $M$. (A hardy reader can skip the examples and study sections 4.7 and 4.8 directly.) Our conclusions list various physical and mathematical applications of our theory as well as speculations on its generalizations, especially to manifolds with additional structures, such as a Kähler one, a possible associative algebraic reformulation of our algebra, the rôle of the Jacobi group and novel Casimir operators, applications to higher spins and gauged versions of the model, extensions of the algebra to $\operatorname{osp}(2 p+2 \mid Q)$ by including the inverse Lichnerowicz wave operator, and finally the model's quantum mechanical 
spectrum.

Tom Branson knew of Lichnerowicz's work.

He may have liked this paper, so it's dedicated to him.

\section{The Model}

\subsection{Orthosymplectic Superalgebras}

The Lie superalgebra $o s p(2 p \mid Q)$ is defined by even supermatrices

$$
\lambda=\left(\begin{array}{l|l}
A & B \\
\hline C & D
\end{array}\right),
$$

subject to the orthosymplectic condition

$$
\lambda J=-J \lambda^{s t},
$$

where the supertranspose is defined by

$$
\left(\begin{array}{c|c}
A & B \\
\hline C & D
\end{array}\right)^{s t} \equiv\left(\begin{array}{c|c}
A^{t} & C^{t} \\
\hline-B^{t} & D^{t}
\end{array}\right) .
$$

The invariant tensor/metric $J$ is given by

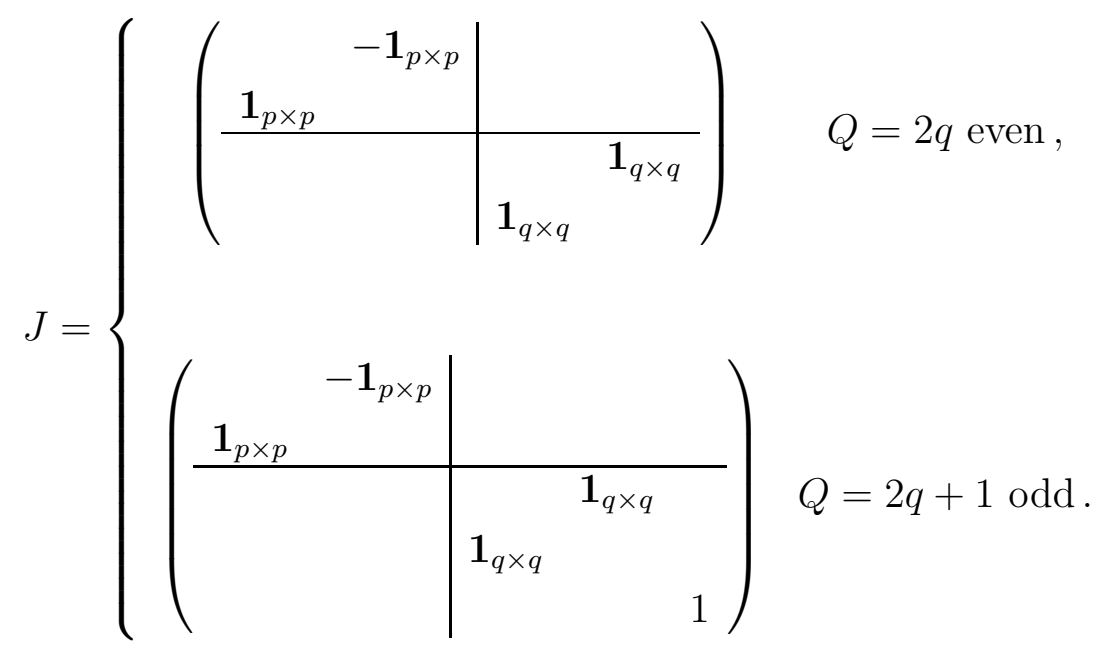


The condition (7) is more simply formulated in terms of $\Lambda \equiv \lambda J$,

$$
\Lambda^{s t}=-J^{2} \Lambda, \quad J^{2}=\operatorname{diag}(\overbrace{-1, \ldots,-1}^{2 p \text { times }}, \overbrace{1, \ldots, 1}^{Q \text { times }}),
$$

whose solution is

$$
\Lambda \equiv\left(\Lambda^{\alpha \beta}\right)=\left(\begin{array}{c|c}
\lambda_{S} & \epsilon \\
\hline \epsilon^{t} & \lambda_{A}
\end{array}\right)
$$

with $\lambda_{S}$ and $\lambda_{A}$ being $2 \times 2$ symmetric and antisymmetric bosonic matrices while $\epsilon$ is a $2 \times 2$ fermionic matrix. The superindices $\alpha, \beta, \ldots$ run over $2 p$ bosonic and $Q$ fermionic values. Note that transformations

$$
\delta X_{\alpha}=J_{\beta \alpha} \Lambda^{\gamma \beta} X_{\gamma},
$$

leave invariant the orthosymplectic inner product

$$
X \cdot Y \equiv-X_{\alpha} J^{\beta \alpha} Y_{\beta}
$$

In addition to time translation invariance, our model will enjoy $Q(2 p+1)$ supersymmetries as well as bosonic $s p(2 p) \ltimes \mathbb{R}^{2 p}$ and $s o([Q / 2],[Q / 2+1 / 2])$ symmetries. These will correspond to $\operatorname{osp}(2 p \mid Q)$ and its fundamental representation $\mathbb{R}^{2 p \mid Q}$. The $\operatorname{osp}(2 p \mid Q)$ generators obey the Lie superalgebra

$$
\left[f_{\alpha \beta}, f_{\gamma \delta}\right\}=4 J_{(\beta(\gamma} f_{\alpha] \delta]},
$$

where the superbracket $[\cdot, \cdot\}$ is a commutator unless both entries are femionic, in which case it equals the anticommutator. We will also often need the quadratic $\operatorname{osp}(2 p \mid Q)$ Casimir

$$
\mathbf{c}=\frac{1}{2} J^{\beta \alpha} f_{\alpha \gamma} J^{\delta \gamma} f_{\delta \beta}
$$

It commutes with all the $\operatorname{osp}(2 p \mid Q)$ generators $f_{\alpha \beta}$.

\subsection{Orthosymplectic Spinning Particle}

The field content of our quantum mechanical theory consists of the embedding coordinates $x^{\mu}$,

$$
x^{\mu}: \mathbb{R} \longrightarrow M
$$


taking values in a $\left(\operatorname{dim}_{M}=d\right)$-dimensional Riemannian target manifold $\left(M, g_{\mu \nu}\right)$, along with $2 p+Q$ additional fields $X^{\mu}$ describing spinning degrees of freedom

$$
X_{\alpha}^{\mu}=\left\{\begin{array}{cc}
\left(\left(\varphi_{1, \ldots, p}^{\mu}\right)^{*}, \varphi_{1, \ldots, p}^{\mu},\left(\psi_{1, \ldots, q}^{\mu}\right)^{*}, \psi_{1, \ldots, q}^{\mu}\right), & Q=2 q \text { even }, \\
\left(\left(\varphi_{1, \ldots, p}^{\mu}\right)^{*}, \varphi_{1, \ldots, p}^{\mu},\left(\psi_{1, \ldots, q}^{\mu}\right)^{*}, \psi_{1, \ldots, q}^{\mu}, \psi^{\mu}\right), & Q=2 q+1 \text { odd } .
\end{array}\right.
$$

The $\varphi$ 's are bosonic and $\psi$ 's fermionic and together $X_{\alpha}^{\mu}$ transforms as the fundamental representation of $\operatorname{osp}(2 p, Q)$ (as in (12)). The action is the natural generalization of the $\mathcal{N}=2$ real supermultiplet

$$
S=\frac{1}{2} \int d t\left\{\dot{x}^{\mu} g_{\mu \nu} \dot{x}^{\nu}+i X_{\alpha}^{\mu} J^{\beta \alpha} \frac{D X_{\beta \mu}}{d t}+\frac{1}{4} R_{\mu \nu \rho \sigma} X_{\alpha}^{\mu} J^{\beta \alpha} X_{\beta}^{\nu} X_{\gamma}^{\rho} J^{\delta \gamma} X_{\delta}^{\sigma}\right\} .
$$

As already mentioned, there are clearly $p(2 p+1)+\frac{1}{2} Q(Q-1)$ bosonic and $2 p Q$ fermionic internal symmetries obtained by $X_{\alpha}^{\mu}$ transformations

$$
\delta X_{\alpha}^{\mu}=J_{\beta \alpha} \Lambda^{\gamma \beta} X_{\gamma}^{\mu}
$$

These hold for any background metric $g_{\mu \nu}$ and we discuss their interpretation later. Less trivial are $Q$ further supersymmetries plus their $2 p$ bosonic "partner" symmetries given by

$$
\begin{aligned}
\delta x^{\mu} & =i X_{\alpha}^{\mu} \varepsilon^{\alpha}, \\
\mathcal{D} X_{\alpha}^{\mu} & =\dot{x}^{\mu} \varepsilon_{\alpha} .
\end{aligned}
$$

In the above, $\mathcal{D}$ and $D / d t$ are the covariant variation and worldline derivatives defined on vectors $v^{\mu}$ by

$$
\begin{aligned}
& \mathcal{D} v^{\mu} \equiv \delta v^{\mu}+\Gamma_{\rho \sigma}^{\mu} \delta x^{\rho} v^{\sigma}=\delta x^{\rho} D_{\rho} v^{\mu}, \\
& \frac{D v^{\mu}}{d t} \equiv \dot{v}^{\mu}+\Gamma_{\rho \sigma}^{\mu} \dot{x}^{\rho} v^{\sigma}=\dot{x}^{\rho} D_{\rho} v^{\mu} .
\end{aligned}
$$

In particular note that their commutator is

$$
\left[\mathcal{D}, \frac{D}{d t}\right] v^{\mu}=\delta x^{\rho} \dot{x}^{\sigma} R_{\rho \sigma}{ }^{\mu}{ }_{\nu} v^{\nu}
$$

To determine whether the variations (20) are symmetries is straightforward upon noting that $\delta\left(v_{\mu} w^{\mu}\right)=v_{\mu} \mathcal{D} w^{\mu}+\left(\mathcal{D} v^{\mu}\right) w_{\mu}$. For the simplest $\mathcal{N}=1$ 
version of the model with $(2 p, Q)=(0,1)$, the four point coupling to the Riemann tensor in the action (18) is absent by virtue of the first Bianchi identity

$$
R_{[\mu \nu \rho] \sigma}=0,
$$

and terms proportional to three $X$ 's (fermions) cancel for the same reason. Therefore the $(0,1)$ model is invariant in any background $M$. For higher cases the terms in the action varying into three $X$ 's cancel by a conspiracy between the four and two-point couplings of these fields. There remain variations proportional to five $X$ 's. For the $\mathcal{N}=2$ model with $(2 p, Q)=(0,2)$, these cancel by the second Bianchi identity

$$
D_{[\kappa} R_{\mu \nu] \rho \sigma}=0,
$$

so this model is also invariant in any background. So far we have just recovered existing results. For general values of $(2 p, Q)$ the obstruction to terms quintic in $X_{\alpha}^{\mu}$ is

$$
\delta S=\frac{i}{8} \int d t X^{\mu} \cdot X^{\nu} X^{\rho} \cdot X^{\sigma} D_{\kappa} R_{\mu \nu \rho \sigma} X^{\kappa} \cdot \varepsilon .
$$

Hence, this theory is supersymmetric on manifolds of covariantly constant curvature

$$
D_{\kappa} R_{\mu \nu \rho \sigma}=0
$$

or in other words, locally symmetric spaces.

\section{Quantization}

\subsection{Canonical Analysis}

To analyze the canonical structure of the theory it is best to work in Darboux coordinates which are found by introducing the vielbein $e_{\mu}{ }^{m}$ and writing the action in terms of $X_{\alpha}^{m} \equiv e_{\mu}^{m} X_{\alpha}^{\mu}$

$$
S=\frac{1}{2} \int d t\left\{\dot{x}^{\mu} g_{\mu \nu} \dot{x}^{\nu}-i X^{m} \cdot \frac{D X_{m}}{d t}+\frac{1}{4} R_{m n r s} X^{m} \cdot X^{n} X^{r} \cdot X^{s}\right\},
$$

where $D V_{m} / d t \equiv \dot{V}_{m}+\dot{x}^{\mu} \omega_{\mu m}{ }^{n} V_{n}$. Then we cast this action into first order form

$$
S^{(1)}=\int d t\left\{p_{\mu} \dot{x}^{\mu}-\frac{i}{2} X_{m} \cdot \dot{X}^{m}-\frac{1}{2} \pi_{\mu} g^{\mu \nu} \pi_{\nu}+\frac{1}{8} R_{m n r s} X^{m} \cdot X^{n} X^{r} \cdot X^{s}\right\},
$$


where the covariant canonical momentum is defined by

$$
\pi_{\mu} \equiv p_{\mu}+\frac{i}{2} \omega_{\mu m n} X^{m} \cdot X^{n}
$$

Since the symplectic form is canonical we immediately quantize by demanding the operator relations ${ }^{3}$

$$
\left[p_{\mu}, x^{\nu}\right]=-i \delta_{\mu}^{\nu}, \quad\left[X_{\alpha}^{m}, X_{\beta}^{n}\right\}=J_{\alpha \beta} \eta^{m n} .
$$

The quantum Hamiltonian is

$$
\begin{aligned}
H & =\frac{1}{2} \pi_{m} \pi^{m}-\frac{1}{8} R_{m n r s} X^{m} \cdot X^{n} X^{r} \cdot X^{s} \\
& +\frac{i}{2} \omega_{m n}{ }^{n} \pi^{m}-\frac{1}{16} \delta_{2 p+Q, 1} R
\end{aligned}
$$

where we have made a definite choice of operator ordering reflected by the terms proportional to a naked spin connection and the scalar curvature. These ensure that the operator $H$ is central ${ }^{4}$ and can be geometrically interpreted as a generalized Laplace operator ${ }^{5}$. Note that we employ the operator ordering $\pi_{m} \equiv e^{\mu}{ }_{m} \pi_{\mu}$.

\subsection{States}

To study the Hilbert space, we view the operators $X_{\alpha}^{\mu}$ as bosonic and fermionic oscillators

$$
X_{\alpha}^{m}=\left\{\begin{aligned}
\left(a_{1, \ldots, p}^{m \dagger}, a_{1, \ldots, p}^{m}, b_{1, \ldots, q}^{m \dagger}, b_{1, \ldots, q}^{m}\right), & Q=2 q \text { even } \\
\left(a_{1, \ldots, p}^{m \dagger}, a_{1, \ldots, p}^{m}, b_{1, \ldots, q}^{m \dagger}, b_{1, \ldots, q}^{m}, b^{m}\right), & Q=2 q+1 \text { odd }
\end{aligned}\right.
$$

\footnotetext{
${ }^{3}$ Unitarity of our quantum mechanical model requires signature $\left(\eta_{m n}\right)=$ $\operatorname{diag}(+1, \ldots,+1)$, but for the study of algebras of differential-geometric operators on semiRiemannian manifolds this requirement can be relaxed. Needless to say, therefore, all our results are germane to an arbitrary choice of signature.

${ }^{4}$ To be sure, throughout this paper we refer to an operator as being central, when it commutes with all the conserved charges of the underlying quantum mechanical system. The prototypical example being the Hamiltonian.

${ }^{5}$ The term proportional to the scalar curvature vanishes for all models save $(2 p, Q)=$ $(0,1)$. Whenever the Riemann tensor is covariantly constant, multiplication by the scalar curvature is obviously a central operation. However, for the $\operatorname{osp}(0,1), \mathcal{N}=1$ supersymmetric model this term ensures that $H$ is central in arbitrary Riemannian backgrounds.
} 
with

$$
\begin{gathered}
{\left[a_{i}^{m}, a_{j n}^{\dagger}\right]=\delta_{i j} \delta_{n}^{m}, \quad\left\{b_{a}^{m}, b_{b n}^{\dagger}\right\}=\delta_{a b} \delta_{n}^{m},} \\
\left\{b^{m}, b^{n}\right\}=\eta^{m n} .
\end{gathered}
$$

The definition of the vacuum state depends on whether $Q$ is even or odd. In the even case, $Q=2 q$ we introduce the Fock vacuum

$$
a_{i}^{\mu}|0\rangle=0=b_{a}^{\mu}|0\rangle
$$

Excited states then correspond to "multi-symmetric tensor-multi forms"

$$
\begin{aligned}
& \left|\Phi_{\mu_{1}^{1} \ldots \mu_{s_{1}}^{1}, \ldots, \mu_{1}^{p} \ldots \mu_{s_{p}}^{p} ; \nu_{1}^{1} \ldots \nu_{k_{1}}^{1}, \ldots, \nu_{1}^{q} \ldots \nu_{k_{q}}^{q}}(x)\right\rangle= \\
& \Phi_{\mu_{1}^{1} \ldots \mu_{s_{1}}^{1}, \cdots, \mu_{1}^{p} \ldots \mu_{s_{p}}^{p} ; \nu_{1}^{1} \ldots \nu_{k_{1}}^{1}, \ldots, \nu_{1}^{q} \ldots \nu_{k_{q}}^{q} \times} \times \\
& \quad a_{1}^{\mu_{1}^{1} \dagger} \ldots a_{1}^{\mu_{s_{1}}^{1} \dagger} \ldots a_{p}^{\mu_{1}^{p} \dagger} \ldots a_{p}^{\mu_{s p}^{p} \dagger} b_{1}^{\nu_{1}^{1} \dagger} \ldots b_{1}^{\nu_{k_{1}}^{1} \dagger} \ldots b_{q}^{\nu_{1}^{q} \dagger} \ldots b_{q}^{\nu_{k_{q}}^{q} \dagger}|0\rangle .
\end{aligned}
$$

When $p=0$ and $q=1$ these are simply differential forms, while for $p=1$, $q=0$ they are just totally symmetric tensors. Moreover, although tensors of the above symmetry type are not irreducible $s o(d)$ representations, any tensor field on the target space manifold can be represented this way. Although, these formulæ look complicated, we will rarely need them, and instead can rely on our supersymmetric quantum mechanical system to provide dynamics and a simple operator algebra on spinning space.

When $Q=2 q+1$ is odd, we need to consider vacua for the algebra $\left\{b^{m}, b^{n}\right\}=\delta^{m n}$ which are now degenerate. As the bilinear operators $b^{[m} b^{n]}$ obey the Lorentz/rotation algebra, vacuum states $|R\rangle$ are labeled by a representation $R$ of $s o(d)$. Moreover, since $\gamma^{m} \equiv \sqrt{2} b^{m}$ obey the Dirac gamma matrix algebra, $R$ must be a spinor representation $|\alpha\rangle$, i.e.,

$$
b^{m}|\alpha\rangle=\frac{1}{\sqrt{2}} \gamma_{\beta}^{m \alpha}|\beta\rangle \text {. }
$$

The precise choice of representation labeled by the spinor index $\alpha$ (not to be confused with the orthosymplectic indices carrying the same name) depends on the dimensionality of the target space. Requiring

$$
a_{i}^{\mu}|\alpha\rangle=0=b_{a}^{\mu}|\alpha\rangle
$$


excited states are then "multi-symmetric tensor-spinor-multi forms"

$$
\begin{aligned}
\left|\Phi_{\mu_{1}^{1} \ldots \mu_{s_{1}}^{1}, \cdots, \mu_{1}^{p} \ldots \mu_{s_{p}}^{p} ; \nu_{1}^{1} \ldots \nu_{k_{1}}^{1}, \cdots, \nu_{1}^{q} \ldots \nu_{k_{q}}^{q}}^{\alpha}(x)\right\rangle= & \\
& \Phi_{\mu_{1}^{1} \ldots \mu_{s_{1}}^{1}, \cdots, \mu_{1}^{p} \ldots \mu_{s_{p}}^{p} ; \nu_{1}^{1} \ldots \nu_{k_{1}}^{1}, \cdots, \nu_{1}^{q} \ldots \nu_{k_{q}}^{q} \times} \times \\
& a_{1}^{\mu_{1}^{1} \dagger} \ldots a_{1}^{\mu_{s_{1}}^{1} \dagger} \ldots a_{p}^{\mu_{1}^{p} \dagger} \ldots a_{p}^{\mu_{s p}^{p} \dagger} b_{1}^{\nu_{1}^{1} \dagger} \ldots b_{1}^{\nu_{k_{1}}^{1} \dagger} \ldots b_{q}^{\nu_{1}^{q} \dagger} \ldots b_{q}^{\nu_{k_{q}}^{q} \dagger}|\alpha\rangle .
\end{aligned}
$$

Let us denote states such as (35) and (38) simply by $|\Phi\rangle$.

In both the $Q$ even and odd cases, by demanding the vacuum to be translation invariant

$$
p_{\mu}|0\rangle=0=p_{\mu}|\alpha\rangle,
$$

we find that the operator $i \pi_{\mu}$ corresponds to the covariant derivative

$$
i \pi_{\mu}|\Phi\rangle=D_{\mu}|\Phi\rangle \text {. }
$$

Similarly, the first two terms of the Hamiltonian are proportional to the Laplacian $\Delta=D_{\mu} D^{\mu}$ so that

$$
H|\Phi\rangle=\left(-\frac{1}{2} \Delta-\frac{1}{8} R_{m n r s} X^{m} \cdot X^{n} X^{r} \cdot X^{s}-\frac{1}{16} \delta_{2 p+Q, 1} R\right)|\Phi\rangle .
$$

Note that in this notation it is important to distinguish between states $D_{\mu}|\Phi\rangle$ and $\left|D_{\mu} \Phi\right\rangle$. For example, when $(2 p, Q)=(0,2)$, the state $\left|\omega_{\mu \nu}\right\rangle=$ $b_{1}^{\mu \dagger} b_{1}^{\nu \dagger} \omega_{\mu \nu}|0\rangle$ is a two-form, while $\left|D_{\rho} \omega_{\mu \nu}\right\rangle=b_{1}^{\mu \dagger} b_{1}^{\nu \dagger} b_{1}^{\rho \dagger} \partial_{\mu} \omega_{\nu \rho}|0\rangle$ is its three-form exterior derivative which does not equal $D_{\mu}\left|\omega_{\nu \rho}\right\rangle=\left(D_{\mu} \omega_{\nu \rho}\right) b_{1}^{\nu \dagger} b_{1}^{\rho \dagger}|0\rangle$. In fact, it is best to think of $|\cdot\rangle$ as a machine which takes as input any tensor from the target space manifold and outputs states such as (35) or (38).

\subsection{Conserved Charges}

To analyze the algebra of conserved charges corresponding to the symmetries $^{6}(19)$ and (20), we note the identities

$$
\begin{aligned}
{\left[\pi_{m}, \pi_{n}\right] } & =\frac{1}{2} R_{m n r s} X^{r} \cdot X^{s}+2 i \omega_{[m n]}{ }^{r} \pi_{r}, \\
{\left[\pi_{m}, X_{\alpha}^{n}\right] } & =i \omega_{m}{ }^{n} X_{\alpha}^{r}, \\
{\left[\pi_{m}, x^{\mu}\right] } & =-i e^{\mu}{ }_{m} .
\end{aligned}
$$

\footnotetext{
${ }^{6}$ We do not consider possible additional symmetries that could arise for backgrounds with special geometries. An excellent starting point for this important investigation are the spinning particle studies $[10,11]$.
} 
Also notice that the operators

$$
M^{m n} \equiv-X^{[m} \cdot X^{n]},
$$

generate the rotation algebra of the tangent space

$$
\left[M^{m n}, M^{r s}\right]=M^{m s} \eta^{n r}-M^{n s} \eta^{m r}+M^{n r} \eta^{m s}-M^{m s} \eta^{n s} .
$$

The conserved charges for time translations, the generalized "supersymmetries" (20), and internal symmetries (19) are readily computed and respectively given by

$$
\begin{aligned}
H & =\frac{1}{2} \pi_{m} \pi^{m}-\frac{1}{8} R_{m n r s} X^{m} \cdot X^{n} X^{r} \cdot X^{s} \\
& +\frac{i}{2} \omega_{m n}{ }^{n} \pi^{m}-\frac{1}{16} \delta_{2 p+Q, 1} R, \\
v_{\alpha} & =i X_{\alpha}^{m} \pi_{m}, \\
f_{\alpha \beta} & =X_{(\alpha}^{m} \eta_{m n} X_{\beta]}^{n} .
\end{aligned}
$$

Again, these are quantum results whose orderings are important (in particular the terms on the second line of the Hamiltonian $H$ are higher order in $\hbar$ and are not needed for classical Poisson brackets). Checking that these charges commute with the Hamiltonian, is tedious but straightforward using the above identities.

When the $\operatorname{osp}(2 p \mid Q)$ fundamental superindex $\alpha$ is odd, the operators $v_{\alpha}$ are "standard" supersymmetry generators rotating bosons $x^{\mu}$ into fermions, and fermions $X_{\alpha}^{m}$ into momenta times bosons. Otherwise, they generate novel bosonic symmetries, which geometrically correspond to gradient and divergence-like operations. To compute their algebra, it is useful to note further identities

$$
\begin{aligned}
{\left[\pi_{m}, \varphi^{\#}\right] } & =-i D_{m} \varphi^{\#} \\
{\left[X_{\alpha}^{m}, \varphi^{\#}\right] } & =-2 \varphi^{m}{ }_{s} X_{\alpha}^{s}, \\
{\left[v_{\alpha}, \varphi^{\#}\right] } & =X_{\alpha}^{m} D_{m} \varphi^{\#}+2 i \varphi_{r s} X_{\alpha}^{r} \pi^{s},
\end{aligned}
$$

where $\varphi_{r s}(x)=-\varphi_{s r}(x)$ and is otherwise arbitrary. Also, we denote the contraction of $X^{m} \cdot X^{n}$ on an antisymmetric tensors by \#, so

$$
\varphi^{\#} \equiv \varphi_{r s} X^{r} \cdot X^{s} .
$$


It also helps to note that

$$
\begin{aligned}
{\left[v_{\alpha}, \pi_{n}\right] } & =\frac{i}{2} X_{\alpha}^{m} R_{m n}^{\#}-X_{\alpha}^{m} \omega_{m n r} \pi^{r}, \\
{\left[v_{\alpha}, X_{\beta}^{n}\right\} } & =i J_{\alpha \beta} \pi^{n}-\omega_{m}{ }^{n}{ }_{r} X_{\alpha}^{m} X_{\beta}^{r} .
\end{aligned}
$$

Detailed yet standard computations yield the commutators between the Hamiltonian and the generators $v_{\alpha}, f_{\alpha \beta}$ which on any Riemannian manifold obey the superalgebra:

$$
\begin{gathered}
{\left[v_{\alpha}, v_{\beta}\right\}=J_{\alpha \beta} \Delta-\frac{1}{2} X_{\alpha}^{m} X_{\beta}^{n} R_{m n}^{\#},} \\
{\left[f_{\alpha \beta}, v_{\gamma}\right\}=2 v_{(\alpha} J_{\beta] \gamma},} \\
{\left[f_{\alpha \beta}, f_{\gamma \delta}\right\}=4 J_{(\beta(\gamma} f_{\alpha] \delta]},} \\
{\left[\square, f_{\alpha \beta}\right]=0 .}
\end{gathered}
$$

In anticipation of its rôle in geometry as the Laplacian, we have defined the operator

$$
\Delta \equiv-2 H-\frac{1}{4} R^{\# \#}-\frac{1}{8} \delta_{2 p+Q, 1} R .
$$

Importantly the "Lichnerowicz wave operator"

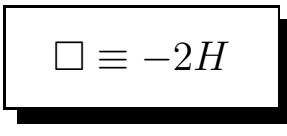

is central, i.e.,

$$
\left[\square, v_{\alpha}\right]=0
$$


in symmetric space backgrounds. Moreover, we denote "supersymmetrization" with unit weight over a pair of superindices by $(\cdot \cdot]$. Since the right hand side of the $[v, v\}$-supercommutator is $J_{\alpha \beta} \square+$ (curvatures), the above relations constitute a finite dimensional super Lie algebra in flat backgrounds. This algebra is the central result of the paper, because it implies a beautiful operator superalgebra on arbitrary tensors on any manifold $M$. Its analysis and geometric interpretation is the subject of the next section.

\section{Geometry}

\subsection{Dirac Operators and $\mathcal{N}=1$ Supersymmetry}

In general, for $Q$ odd, the fermionic operator

$$
v_{2 p+Q}=b^{m} \pi_{m}=\frac{1}{\sqrt{2}} \not D,
$$

acts on states as the Dirac operator. Moreover, at $(2 p, Q)=(0,1)$, there are no bosonic oscillators and a single fermionic one $b^{m}=\frac{1}{\sqrt{2}} \gamma^{m}$ corresponding to the Dirac matrices, so we have conserved charges $H$ and $v_{1}=\frac{1}{\sqrt{2}} \not D$ and no $f_{\alpha \beta}$ 's as the internal symmetry group $\operatorname{osp}(0 \mid 1)$ is empty. Their algebra is just the $\mathcal{N}=1$ supersymmetry algebra

$$
\not D^{2}=-2 H=\Delta+\frac{1}{4} R \equiv \square,
$$

or in terms of geometry, the Weitzenbock identity for the square of the Dirac operator in curved space. Since the Laplace-like operator $\square$ commutes with the Dirac operator $\not D$, we may view it as a Lichnerowicz wave operator.

\subsection{Differential Forms and $\mathcal{N}=2$ Supersymmetry}

At $(2 p, Q)=(0,2)$, we have a pair of fermionic oscillators $\left(b^{m \dagger}, b^{m}\right)$. Identifying $X_{1}^{\mu}=b^{\mu \dagger}=d x^{\mu}$ with anticommuting coordinate differentials $d x^{\mu}$, the operator

$$
v_{1}=\mathbf{d},
$$


the exterior derivative, while states are differential forms. Then we identify

$$
v_{2}=\boldsymbol{\delta}
$$

with the codifferential and the algebra (49) gives

$$
\{\mathbf{d}, \boldsymbol{\delta}\}=-2 H=\Delta+\frac{1}{4} R^{\# \#} \equiv \square .
$$

The right hand side, $\square=D_{\mu} D^{\mu}+\frac{1}{4} R^{\# \#}$ equals the form Laplacian, which is the central modification of the usual Laplace operator. Again, we may view it as a Lichnerowicz wave operator acting on differential forms. The internal $o s p(0 \mid 2) \cong s o(1,1)$ algebra has a single generator

$$
f_{12}=b^{m \dagger} b_{m}-\frac{1}{2} \operatorname{dim}_{M}
$$

The operator

$$
\mathbf{N} \equiv b^{m \dagger} b_{m}
$$

acts on states (= forms) by counting their degree (or number of indices). Hence, we have an $u(1 \mid 1)$ superalgebra

$$
\begin{gathered}
\{\mathbf{d}, \boldsymbol{\delta}\}=\square, \quad[\square, \mathbf{d}]=0=[\square, \boldsymbol{\delta}], \\
{[\mathbf{N}, \mathbf{d}]=\mathbf{d}, \quad[\mathbf{N}, \boldsymbol{\delta}]=-\boldsymbol{\delta}, \quad[\mathbf{N}, \Delta]=0 .}
\end{gathered}
$$

Alternately, the $u(1 \mid 1)$ superalgebra of the $\mathcal{N}=2$ real multiplet model can be reinterpreted as a central extension by $\square$ of the semidirect product of superalgebras $\operatorname{osp}(0 \mid 2) \ltimes \mathbb{R}^{0 \mid 2}$, where $f_{\alpha \beta} \equiv J_{\alpha \beta} \mathbf{N}$ generates the $\operatorname{osp}(0 \mid 2)$ factor.

\subsection{Symmetric Algebras and Quantum Mechanics}

The above discussion relating the $\operatorname{osp}(0 \mid 1)$ and $\operatorname{ssp}(0 \mid 2)$ models to geometry reviewed known results. We now turn to the purely bosonic ${ }^{7}$ osp $(2 \mid 0)$ model, and begin our presentation of new material.

\footnotetext{
${ }^{7}$ Spinning particle models with "commuting-spinor" coordinates have appeared before in the literature [22].
} 
Lichnerowicz introduced a modified Laplacian $\Delta^{(n)}$ acting on $n$-index totally symmetric tensors [6] in order to facilitate a computation of the massive spin 2 propagator on spaces of constant curvature ${ }^{8}$

$$
R_{\mu \nu}^{\rho \sigma}=-\frac{2 R}{d(d-1)} \delta_{[\mu}^{\rho} \delta_{\nu]}^{\sigma} .
$$

(We shall employ units $R=-d(d-1)$ whenever working with constant curvature spaces. Factors of $R$ can be reinstated by inserting appropriate powers of $1=-\frac{R}{d(d-1)}$ on the grounds of dimensionality. All formulæ obtained this way are valid for spaces of positive or negative scalar curvature. The physical cosmological constant, $\Lambda=-R / d$.) Low lying examples of this Lichnerowicz wave operator are

$$
\begin{aligned}
\Delta^{(0)} \varphi & =\Delta \varphi, \\
\Delta^{(1)} \varphi_{\mu} & =(\Delta-d+1) \varphi_{\mu}, \\
\Delta^{(2)} \varphi_{\mu \nu} & =(\Delta-2 d) \varphi_{\mu \nu}+2 g_{\mu \nu} \varphi_{\rho}{ }^{\rho}, \\
\Delta^{(3)} \varphi_{\mu \nu \rho} & =(\Delta-3 d-3) \varphi_{\mu \nu \rho}+6 g_{(\mu \nu} \varphi_{\rho) \sigma}{ }^{\sigma},
\end{aligned}
$$

Formulæ for general $n$ are known (see [8] and also [23, 24]) but are also a direct consequence of the models given here, in particular the generalization to an arbitrary symmetric space is

$$
\Delta^{(n)} \varphi_{\mu_{1} \ldots \mu_{n}}=\Delta \varphi_{\mu_{1} \ldots \mu_{n}}+n(n-1) R_{\left(\mu_{1}\right.}{ }^{\rho} \mu_{2}{ }^{\sigma} \varphi_{\left.\mu_{3} \ldots \mu_{n}\right) \rho \sigma}+n R_{\rho\left(\mu_{1}\right.} \varphi_{\left.\mu_{2} \ldots \mu_{n}\right)}{ }^{\rho} .
$$

Here, $\Delta=D_{\mu} D^{\mu}$ is the usual Laplacian, the key point being that $\Delta^{(n)}$ "commutes" with symmetrized trace, metric, divergence and gradient operations

$$
\begin{aligned}
g^{\mu \nu} \Delta^{(n)} \varphi_{\mu \nu \rho_{3} \ldots \rho_{n}} & =\Delta^{(n-2)} \varphi_{\mu \rho_{3} \ldots \rho_{n}}^{\mu}, \\
\Delta^{(n+2)} g_{\left(\rho_{1} \rho_{2}\right.} \varphi_{\left.\rho_{3} \ldots \rho_{n+2}\right)} & =g_{\left(\rho_{1} \rho_{2}\right.} \Delta^{(n)} \varphi_{\left.\rho_{3} \ldots \rho_{n+2}\right)}, \\
D^{\mu} \Delta^{(n)} \varphi_{\mu \rho_{2} \ldots \rho_{n}} & =\Delta^{(n-1)} D^{\mu} \varphi_{\mu \rho_{2} \ldots \rho_{n}}, \\
\Delta^{(n+1)} D_{\left(\rho_{1}\right.} \varphi_{\left.\rho_{2} \ldots \rho_{n+1}\right)} & =D_{\left(\rho_{1}\right.} \Delta^{(n)} \varphi_{\left.\rho_{2} \ldots \rho_{n+1}\right)} .
\end{aligned}
$$

\footnotetext{
${ }^{8}$ Our Ricci curvature convention is $R_{\mu \nu}=R_{\rho \mu \nu}{ }^{\rho}$.
} 
Indeed, the Lichnerowicz wave operator $\Delta^{(n)}$ corresponds to $-2 H$, the Hamiltonian of our quantum mechanical system at $(2 p, Q)=(2,0)$. To see this relation we recall the symmetric algebra formalism of [8] where the operators $(\mathbf{N}, \mathbf{t r}, \mathbf{g}, \mathbf{d i v}, \mathbf{g r a d}, \square)$ acting on symmetric tensors were defined:

\begin{tabular}{c|c|c}
\hline Index & $\mathbf{N}=d x^{\mu} \partial_{\mu}$ & Counts indices. \\
Trace & $\mathbf{t r}=g^{\mu \nu} \partial_{\mu} \partial_{\nu}$ & Traces a pair of indices. \\
Metric & $\mathbf{g}=g_{\mu \nu} d x^{\mu} d x^{\nu}$ & Multiplies by the metric and symmetrizes. \\
Divergence & $\mathbf{d i v}=g^{\mu \nu} \partial_{\mu} D_{\nu}$ & The covariant divergence. \\
Gradient & $\mathbf{g r a d}=d x^{\mu} D_{\mu}$ & Symmetrized covariant derivative. \\
\hline
\end{tabular}

In that work, commuting symbols $d x^{\mu}$ and $\partial_{\mu} \equiv \partial / \partial\left(d x^{\mu}\right)$ with algebra ${ }^{9}$

$$
\left[\partial_{\mu}, d x^{\nu}\right]=\delta_{\mu}^{\nu},
$$

were employed for index bookkeeping and the algebra operated on sums of symmetric tensors

$$
\Phi=\sum_{n} \varphi_{\mu_{1} \ldots \mu_{n}} d x^{\left(\mu_{1}\right.} \cdots d x^{\left.\mu_{n}\right)} .
$$

Moreover the operators (g, $\mathbf{N}, \mathbf{t r})$ formed an $s p(2)$ Lie algebra with (grad, div) transforming as its fundamental doublet representation. The Lichnerowicz wave operator $\square$ was given by ${ }^{10}$

$$
\square=\Delta+\mathbf{c}+\frac{d(d-4)}{4}=[\operatorname{div}, \operatorname{grad}]+2 \mathbf{c}+\frac{d(d-4)}{2},
$$

where the $s p(2)$ Casimir of (15) equals

$$
\mathbf{c}=\frac{1}{2} J^{\beta \alpha} f_{\alpha \gamma} J^{\delta \gamma} f_{\delta \beta}=\mathbf{g} \mathbf{t r}-\mathbf{N}(\mathbf{N}+d-2)-\frac{d(d-4)}{4} .
$$

The operator $\square$ acts on an $n$-index symmetric tensor by the operator $\Delta^{(n)}$ given above. Importantly, $\square$ is central - commuting with ( $\mathbf{N}, \mathbf{t r}, \mathbf{g}, \mathbf{d i v}, \mathbf{g r a d})$ which explains equations (64) above.

\footnotetext{
${ }^{9}$ We remind the reader that the dual differential $\partial_{\mu}$ corresponds to the quantum mechanical oscillator $a_{\mu}$, so does not act on functions of coordinates such as the metric.

${ }^{10}$ Note that the equivalent relation in equation (30) of [8] is quoted with the wrong sign convention for the cosmological constant relative to the remainder of that work. Also, we have shifted the definition of $\mathbf{c}$ by the constant $d(d-4) / 4$ to conform with the uniform definition (15).
} 
Identifying the Fock space oscillators of the previous section as

$$
d x^{\mu} \leftrightarrow a_{1}^{\mu \dagger}, \quad \frac{\partial}{\partial\left(d x^{\mu}\right)} \leftrightarrow a_{1 \mu}
$$

establishes an isomorphism between the symmetric space algebra and our quantum mechanical system at $(2 p, Q)=(2,0)$. States $|\Phi\rangle$ correspond to symmetric tensors $\Phi=\sum_{s} \phi_{\mu_{1} \ldots \mu_{s}} d x^{\mu_{1}} \ldots d x^{\mu_{s}}$ and the dictionary for conserved charges of the model reads

$$
\begin{aligned}
& \left(f_{\alpha \beta}\right) \leftrightarrow\left(\begin{array}{cc}
\mathbf{g} & \mathbf{N}+\frac{d}{2} \\
\mathbf{N}+\frac{d}{2} & \mathbf{t r}
\end{array}\right) \\
& \left(v_{\alpha}\right) \leftrightarrow \quad\left(\begin{array}{c}
\operatorname{grad} \\
\operatorname{div}
\end{array}\right) \\
& -2 H \leftrightarrow \leftrightarrow \quad \square .
\end{aligned}
$$

Also, the inner product and adjoint operations introduced in [8] correspond to the inner product of quantum mechanical states.

In fact this dictionary even provides a generalization of the symmetric algebra formalism to any symmetric space, rather than simply spaces of constant curvature. For completeness, we write out explicitly the algebra (49)

$$
\begin{aligned}
{[\mathbf{t r}, \mathbf{g}]=} & 4 \mathbf{N}+2 d, \\
{[\operatorname{tr}, \mathbf{g r a d}]=2 \operatorname{div}, } & {[\operatorname{div}, \mathbf{g}]=2 \mathbf{g r a d}, } \\
{[\operatorname{div}, \mathbf{g r a d}]=} & \square-2 R_{\mu \nu \rho \sigma} d x^{\mu} \partial^{\nu} d x^{\rho} \partial^{\sigma}, \\
{[\mathbf{N}, \mathcal{O}] } & =\mathrm{wt}_{\mathcal{O}} \cdot \mathcal{O},
\end{aligned}
$$

where the weights of the index operator $N$ provide a five-grading:

$$
\begin{array}{c|cccccc}
\mathcal{O} & \text { tr } & \text { div } & \mathbf{N} & \square & \text { grad } & \text { g } \\
\hline \text { wt }_{\mathcal{O}} & -2 & -1 & 0 & 0 & 1 & 2
\end{array}
$$

The operators $\{\mathbf{g}, \mathbf{N}, \mathbf{t r}\}$ generate an $s l(2, \mathbb{R})=s p(2)=o s p(2 \mid 0)$ Lie algebra and (grad, div) transform as its fundamental representation. When the curvature vanishes we obtain the Lie algebra of the Jacobi group (a very useful reference is [25]). Otherwise the algebra is a deformation thereof. 


\subsection{Lichnerowicz Dirac Operator}

Our next example is the $\operatorname{osp}(2 \mid 1)$ model. It is the first model with both bosonic and fermionic oscillators and is distinguished among all models by possessing a central, Lichnerowicz-Dirac operator. This operator was first uncovered in a study of partially massless higher spin fermi fields [17]. It displays an extremely interesting "statistical-transmutation" property. Namely, that although it is an odd operator (proportional to odd numbers of Dirac matrices), it commutes with every other operator in the algebra (fermionic or otherwise).

The $\operatorname{osp}(2 \mid 1)$ model has a pair of bosonic oscillators, interpreted as commuting coordinate differentials and their duals

$$
\left(a^{m \dagger}, a_{m}\right) \cong\left(d x^{m}, \partial_{m}\right),
$$

with algebra (65), just as for the $\operatorname{osp}(2 \mid 0)$ model. In addition there is a single vector of fermionic oscillators corresponding to the Dirac matrices

$$
b^{m} \cong \frac{1}{\sqrt{2}} \gamma^{m}
$$

as for the $\operatorname{ssp}(0 \mid 1), \mathcal{N}=1$ supersymmetric model. States are totally symmetric spinor-valued tensors. In addition to the Dirac operator $\not D$ of the $\operatorname{sep}(0 \mid 1)$ model, and operators $(\mathbf{N}, \mathbf{t r}, \mathbf{g}, \mathbf{d i v}, \mathbf{g r a d})$ of the $\operatorname{osp}(2 \mid 0)$ model and their mutual Lichnerowicz operator $\square$, there are an additional pair of operators $\left(\boldsymbol{\gamma}, \boldsymbol{\gamma}^{*}\right)$, which either add a symmetric tensor index carried by a Dirac matrix, or take a gamma-trace ${ }^{11}$ :

$$
\begin{aligned}
\gamma: \psi_{\mu_{2} \ldots \mu_{s}} & \longmapsto \gamma_{\left(\mu_{1}\right.} \psi_{\left.\mu_{2} \ldots \mu_{s}\right)}, \\
\gamma^{*}: \psi_{\mu_{1} \ldots \mu_{s}} & \longmapsto s \gamma^{\mu} \psi_{\mu \mu_{2} \ldots \mu_{s}} .
\end{aligned}
$$

Together, $\left(\mathbf{g}, \boldsymbol{\gamma}, \mathbf{N}, \boldsymbol{\gamma}^{*}, \mathbf{t r}\right)$ generate $\operatorname{osp}(2 \mid 1)$

$$
\left(f_{\alpha \beta}\right)=\left(\begin{array}{cc|c}
\mathbf{g} & \mathbf{N}+\frac{d}{2} & \frac{1}{\sqrt{2}} \gamma \\
\mathbf{N}+\frac{d}{2} & \mathbf{t r} & \frac{1}{\sqrt{2}} \gamma^{*} \\
\hline \frac{1}{\sqrt{2}} \gamma & \frac{1}{\sqrt{2}} \gamma^{*} & 0
\end{array}\right), \quad\left[f_{\alpha \beta}, f_{\gamma \delta}\right\}=4 J_{(\beta(\gamma)} f_{\alpha] \delta]} .
$$

\footnotetext{
${ }^{11}$ Strictly we should write, for example, either $\gamma\left|\psi_{\mu_{2} \ldots \mu_{s}}\right\rangle=\left|\gamma_{\mu_{1}} \psi_{\mu_{2} \ldots \mu_{s}}\right\rangle$ in a quantum mechanical language, or $\gamma \psi_{\mu_{1} \ldots \mu_{s-1}} d x^{\mu_{1}} \cdots d x^{\mu_{s-1}}=\gamma_{\mu_{1}} \psi_{\mu_{2} \ldots \mu_{s}} d x^{\mu_{1}} \cdots d x^{\mu_{s}}$, in a geometry notation.
} 
This superalgebra mostly replicates the one given above for $\operatorname{ssp}(2 \mid 0)$, but in addition $\left(\boldsymbol{\gamma}, \boldsymbol{\gamma}^{*}\right)$ transform as an $s p(2)$ doublet:

$$
\begin{gathered}
{[\mathbf{g}, \boldsymbol{\gamma}]=0 \quad=\left[\boldsymbol{\gamma}^{*}, \mathbf{t r}\right],} \\
{\left[\boldsymbol{\gamma}^{*}, \mathbf{g}\right]=2 \boldsymbol{\gamma},} \\
{[N, \boldsymbol{t r}, \boldsymbol{\gamma}]=2 \boldsymbol{\gamma}^{*},} \\
{[\boldsymbol{\gamma}]=\boldsymbol{\gamma}, \quad\left[N, \boldsymbol{\gamma}^{*}\right]=-\boldsymbol{\gamma}^{*},} \\
\{\boldsymbol{\gamma}, \boldsymbol{\gamma}\}=2 \mathbf{g}, \quad\left\{\boldsymbol{\gamma}^{*}, \boldsymbol{\gamma}^{*}\right\}=2 \mathbf{t r}, \\
\left\{\boldsymbol{\gamma}, \boldsymbol{\gamma}^{*}\right\}=2 \mathbf{N}+d .
\end{gathered}
$$

Writing out the second $[f, v\}$ line of the algebra (49) yields (for those commutation relations not given in the previous $\operatorname{osp}(2 \mid 0)$ section)

$$
\begin{gathered}
{\left[\gamma^{*}, \operatorname{grad}\right]=\not D=[\operatorname{div}, \gamma],} \\
{[\not D, \mathbf{g}]=0=[\not D, \mathbf{t r}],} \\
\{\not D, \gamma\}=2 \operatorname{grad}, \quad\left\{\not D, \gamma^{*}\right\}=2 \operatorname{div} .
\end{gathered}
$$

Finally it remains to explicate the $[v, v\}$ relations. Since these are simplest (and most utilizable) for the case of constant curvature, we specialize to

$$
R_{m n r s}=2 \eta_{m[r} \eta_{s] n}
$$

Then we find

$$
\begin{aligned}
{[\not D, \text { grad }] } & =\boldsymbol{\gamma}\left[\mathbf{N}+\frac{d-1}{2}\right]-\mathbf{g} \boldsymbol{\gamma}^{*}, \\
\not D^{2} & =\square-\mathbf{c}-\mathbf{N}+\boldsymbol{\gamma} \boldsymbol{\gamma}^{*}-\frac{1}{8} d(3 d-5), \\
{[\operatorname{div}, \text { grad }] } & =\square-2 \mathbf{c}-\frac{1}{2}\left[\mathbf{N}-\boldsymbol{\gamma} \boldsymbol{\gamma}^{*}\right]-\frac{1}{8} d(3 d-7), \\
{[\operatorname{div}, \not D] } & =\left[\mathbf{N}+\frac{d-1}{2}\right] \boldsymbol{\gamma}^{*}-\boldsymbol{\gamma} \mathbf{t r} .
\end{aligned}
$$

Here

$$
\mathbf{c}=\mathbf{g} \mathbf{t r}-\mathbf{N}(\mathbf{N}+d-1)+\gamma \boldsymbol{\gamma}^{*}-\frac{1}{4} d(d-2),
$$


is the $\operatorname{osp}(2 \mid 1)$ Casimir and the central Lichnerowicz wave operator

$$
=\Delta+\mathbf{c}+\frac{1}{8} d(d-3) .
$$

Incidentally, introducing the supergravity-inspired operators ${ }^{12}$

$$
\mathcal{G} \text { rad }=\operatorname{grad}+\frac{i}{2} \gamma, \quad \operatorname{Div}=\operatorname{div}-\frac{i}{2} \gamma^{*},
$$

the above algebra simplifies to

$$
[\mathcal{D i v}, \mathcal{G} \mathbf{r a d}]=\square-2 \mathbf{c}-\frac{3}{8} d(d-3) .
$$

Armed with the above algebra, we can now search for further central operators, and find a generalized Dirac operator

$$
\mathcal{D}=\gamma \not D \gamma^{*}-\gamma^{*} \not D \gamma
$$

This is the operator introduced in [17]. Although it is clearly of odd Grassmann parity, it commutes with all other operators $(\mathbf{g}, \gamma, \operatorname{grad}, \mathbf{N}, \square, \not D, \operatorname{div}$, $\gamma^{*}, \mathbf{t r}$ ) in constant curvature backgrounds. In particular, it fails to commute with $\not D$ in general symmetric spaces (in which $\square$, being the Hamiltonian of our spinning particle model, is still central). It commutes with the $\operatorname{sep}(2 \mid 1)$ generators in any background and seems to have no generalization to higher $\operatorname{osp}(2 p \mid Q)$ models with $Q$ odd. So to the best of our (current) knowledge its existence is a peculiarity of symmetric spinor-tensors.

\subsection{Multisymmetric Tensors}

When the oscillator algebra is $\operatorname{osp}(2 p \mid 0)=s p(2 p)$, states are tensors whose indices are grouped into totally symmetric subsets

$$
\varphi_{\mu_{1}^{1} \ldots \mu_{s_{1}}^{1}, \ldots, \mu_{1}^{p} \ldots \mu_{s_{p}}^{p}}=\varphi_{\left(\mu_{1}^{1} \ldots \mu_{s_{1}}^{1}\right), \ldots,\left(\mu_{1}^{p} \ldots \mu_{s_{p}}^{p}\right)} \cdot
$$

The oscillators can be viewed as commuting coordinate differentials and their duals

$$
X_{\alpha}^{\mu}=\left\{d x_{1}^{\mu}, \ldots d x_{p}^{\mu}, \partial_{1}^{\mu}, \ldots, \partial_{p}^{\mu}\right\},
$$

\footnotetext{
${ }^{12}$ Recall that $\mathcal{N}=1$ cosmological supergravity is most simply defined in terms of a modified covariant derivative $\mathcal{D}_{\mu}=D_{\mu}+\frac{1}{2} \sqrt{-\Lambda / 3} \gamma_{\mu}[26,27]$. Note also that the algebra presented in section 7 of [8] suffers some typographical errors amounting to interchanging (grad, div $) \leftrightarrow(\mathcal{G} \mathbf{r a d}, \mathcal{D} \mathbf{i v})$. The correct algebra is presented above.
} 
with algebra

$$
\left[\partial_{i \mu}, d x_{j}^{\nu}\right]=\delta_{i j} \delta_{\mu}^{\nu}
$$

In Young diagram notation we could depict the tensor in (86) as

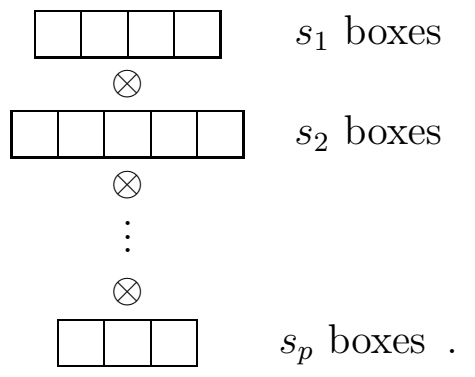

The operations $\mathbf{g}, \mathbf{N}$ and $\mathbf{t r}$ of the above sections correspond to adding a pair of boxes, counting the number of boxes, or removing a pair of boxes in the single row case, respectively. In addition we would now like to count the number of indices in a given row, move boxes from one row to another, and add or remove pairs of boxes from distinct rows. All these operations are achieved by promoting $\mathbf{g}, \mathbf{N}$ and $\operatorname{tr}$ to $p \times p$ matrices of operators:

$$
\begin{aligned}
\mathbf{g} & \equiv\left(\mathbf{g}_{i j}\right)=\left(d x_{i}^{\mu} g_{\mu \nu} d x_{j}^{\nu}\right), \\
\mathbf{N} & \equiv\left(\mathbf{N}_{i j}\right)=\left(d x_{i}^{\mu} \partial_{j \mu}\right), \\
\mathbf{t r} & \equiv\left(\mathbf{t r}_{i j}\right)=\left(\partial_{i \mu} g^{\mu \nu} \partial_{j \nu}\right)
\end{aligned}
$$

These operators correspond precisely to the $p(2 p+1)$ conserved $o s p(2 p \mid 0)$ charges of the underlying quantum mechanical model

$$
\left(f_{\alpha \beta}\right)=\left(\begin{array}{cc}
\mathbf{g} & \mathbf{N}+\frac{d}{2} \mathbf{1} \\
\mathbf{N}^{t}+\frac{d}{2} \mathbf{1} & \mathbf{t r}
\end{array}\right),
$$

where $\mathbf{1}$ is the $p \times p$ identity matrix and the matrices $\mathbf{g}$ and $\mathbf{t r}$ are symmetric. These obey the $s p(2 p)$ Lie algebra following from (49)

$$
\begin{aligned}
{\left[\mathbf{N}_{i j}, \mathbf{g}_{k l}\right] } & =2 \delta_{j(k} \mathbf{g}_{l) i}, \\
{\left[\mathbf{t r}_{i j}, \mathbf{g}_{k l}\right] } & =4 \delta_{(k(i} \mathbf{N}_{l) j}+2 d \delta_{i(k} \delta_{l) j}, \\
{\left[\mathbf{N}_{i j}, \mathbf{t r}_{k l}\right] } & =-2 \delta_{i(k} \mathbf{t r}_{l) j}, \\
{\left[\mathbf{N}_{i j}, \mathbf{N}_{k l}\right] } & =\delta_{j k} \mathbf{N}_{i l}-\delta_{i l} \mathbf{N}_{k j}
\end{aligned}
$$


whose quadratic Casimir (15) now reads

$$
\begin{aligned}
\mathbf{c} & =\operatorname{tr}\left[\frac{\mathbf{g} \mathbf{t r}+\mathbf{t r} \mathbf{g}}{2}-\left(\mathbf{N}+\frac{d}{2} \mathbf{1}\right)^{2}\right] \\
& =\mathbf{g}_{i j} \mathbf{t r}_{i j}-\mathbf{N}_{i j} \mathbf{N}_{j i}-(d-p-1) \mathbf{N}_{i i}-\frac{1}{4} d p(d-2 p-2) .
\end{aligned}
$$

There are now gradient and divergence operators acting on each row

$$
\left(v_{\alpha}\right)=\left(\begin{array}{c}
\operatorname{grad}_{i} \\
\operatorname{div}_{i}
\end{array}\right),
$$

which transform as the fundamental representation of $s p(2 p)$

$$
\begin{array}{ll}
{\left[\mathbf{N}_{i j}, \operatorname{grad}_{k}\right]=\delta_{j k} \operatorname{grad}_{i},} & {\left[\mathbf{N}_{i j}, \operatorname{div}_{k}\right]=-\delta_{i k} \operatorname{div}_{j},} \\
{\left[\operatorname{tr}_{i j}, \operatorname{grad}_{k}\right]=2 \delta_{k(i} \operatorname{div}_{j)},} & {\left[\mathbf{g}_{i j}, \operatorname{div}_{k}\right]=-2 \delta_{k(i} \operatorname{grad}_{j)} .}
\end{array}
$$

They obey the algebra

$$
\begin{aligned}
{\left[\operatorname{grad}_{i}, \operatorname{grad}_{j}\right] } & =R_{\mu \nu \rho \sigma} d x_{i}^{\mu} d x_{j}^{\nu} d x_{k}^{\rho} \partial_{k}^{\sigma} \\
{\left[\operatorname{div}_{i}, \operatorname{grad}_{j}\right] } & =\delta_{i j} \Delta-R_{\mu \nu \rho \sigma} d x_{j}^{\mu} \partial_{i}^{\nu} d x_{k}^{\rho} \partial_{k}^{\sigma}, \\
{\left[\operatorname{div}_{i}, \operatorname{div}_{j}\right] } & =R_{\mu \nu \rho \sigma} \partial_{i}^{\mu} \partial_{j}^{\nu} d x_{k}^{\rho} \partial_{k}^{\sigma} .
\end{aligned}
$$

Moreover, the Lichnerowicz wave operator

$$
\square=\Delta+R_{\mu \nu \rho \sigma} d x_{i}^{\mu} \partial_{i}^{\nu} d x_{k}^{\rho} \partial_{k}^{\sigma},
$$

commutes with $\mathbf{g}, \mathbf{N}$ and $\mathbf{t r}$ on any manifold, and in the case of symmetric spaces also commutes with div and grad. Finally, for constant curvature manifolds (61), the relations (96) and (97) simplify to

$$
\begin{aligned}
& {\left[\operatorname{grad}_{i}, \operatorname{grad}_{j}\right]=2 \mathbf{g}_{k[i} \mathbf{N}_{j] k},} \\
& {\left[\operatorname{div}_{i}, \operatorname{grad}_{j}\right]=\delta_{i j}\left(\square-\mathbf{c}-\frac{1}{4} d p[d-2 p-2]\right)} \\
& -\mathbf{g}_{j k} \mathbf{t r}_{k i}+\mathbf{N}_{j k} \mathbf{N}_{k i}+(d-p-1) \mathbf{N}_{j i}, \\
& {\left[\operatorname{div}_{i}, \operatorname{div}_{j}\right]=-2 \mathbf{N}_{[j|k|} \operatorname{tr}_{i] k}}
\end{aligned}
$$

and

$$
\square=\Delta+\mathbf{c}+\frac{1}{4} d p(d-2 p-2)
$$




\subsection{Multiforms}

Models with $\operatorname{osp}(0 \mid 2 q)$ oscillators have been studied extensively in the literature. The oscillator modes are all fermionic and their bilinears represent the $s o(2 q)$ Lie algebra. In terms of geometry, they correspond to $q$ sets of anticommuting coordinate differentials and their duals $\left\{\left(d x_{1}^{\mu}, \partial_{1 \nu}\right), \ldots,\left(d x_{q}^{\mu}, \partial_{q \nu}\right)\right\}$ subject to

$$
\left\{d x_{a}^{\mu}, \partial_{b \nu}\right\}=\delta_{\nu}^{\mu} \delta_{a b}
$$

with indices $a, b, c, \ldots=1, \ldots, q$. States are therefore multiforms, i.e., tensors with groups of totally antisymmetric indices

$$
\phi_{\nu_{1}^{1} \ldots \nu_{k_{1}}^{1}, \ldots, \nu_{1}^{q} \ldots \nu_{k_{q}}^{q}}=\phi_{\left[\nu_{1}^{1} \ldots \nu_{k_{1}}^{1}\right], \ldots,\left[\nu_{1}^{q} \ldots \nu_{k_{q}}^{q}\right]}
$$

Pictorially, the analog of (89) would be products of columns. Geometric operations on beasts of this type have been studied in detail $[19,20,21]$ in the context of mixed symmetry higher spin fields. Gauged versions of the underlying $S O(2 q)$ spinning particle models and their relation to higher spins appeared in $[28,31,32]$.

Once again the conserved charges $f_{\alpha \beta}, v_{\alpha}$ and $H$ correspond to geometric operators. The $s o(2 q)$ generators,

$$
\left(f_{\alpha \beta}\right)=\left(\begin{array}{cc}
\mathrm{g} & \mathbf{N}-\frac{d}{2} \mathbf{1} \\
-\mathbf{N}^{t}+\frac{d}{2} \mathbf{1} & \mathbf{t r}
\end{array}\right),
$$

either count indices from a set or move an index from one set to another via

$$
\mathbf{N}=\left(b_{a}^{m \dagger} \eta_{m n} b_{b}^{n}\right),
$$

or add or remove a pair of indices from a pair of antisymmetric index sets using the $q \times q$ antisymmetric matrices of operators

$$
\mathbf{g}=\left(b_{a}^{m \dagger} \eta_{m n} b_{b}^{n \dagger}\right)=-\mathbf{g}^{t} \quad \text { or } \quad \mathbf{t r}=\left(b_{a}^{m} \eta_{m n} b_{b}^{n}\right)=-\mathbf{t r}^{t} .
$$

Naturally, it is not possible to trace or add a pair of boxes using the metric on a single column. As an example of these operations, the Riemann tensor 
$R_{\mu \nu \rho \sigma}=R_{[\mu \nu]_{1}[\rho \sigma]_{2}}$ is a biform and

$$
\left(f_{\alpha \beta}\right) R_{\mu \nu \rho \sigma}=\left(\begin{array}{cccc}
0 & g_{[\kappa[\eta} R_{\left.\mu \nu]_{1} \rho \sigma\right]_{2}} & {\left[2-\frac{d}{2}\right] R_{\mu \nu \rho \sigma}} & 2 R_{[\mu \nu \rho]_{1} \sigma} \\
-g_{[\kappa[\eta} R_{\left.\mu \nu]_{1} \rho \sigma\right]_{2}} & 0 & -2 R_{\mu[\nu \rho \sigma]_{2}} & {\left[2-\frac{d}{2}\right] R_{\mu \nu \rho \sigma}} \\
-\left[2-\frac{d}{2}\right] R_{\mu \nu \rho \sigma} & 2 R_{\mu[\nu \rho \sigma]_{2}} & 0 & -4 R_{\mu \rho} \\
-2 R_{[\mu \nu \rho]_{1} \sigma} & -\left[2-\frac{d}{2}\right] R_{\mu \nu \rho \sigma} & 4 R_{\mu \rho} & 0
\end{array}\right)
$$

(for clarity we have labeled index sets by subscripts 1,2 and have not imposed the first Bianchi identity). Clearly, an index notation rapidly becomes cumbersome, and it is best just to think of the operators acting on states, but it should at least make the interpretation of these operators clear. Their so $(2 q)$ algebra follows from (49)

$$
\left[f_{\alpha \beta}, f_{\gamma \delta}\right]=4 J_{[\beta[\gamma} f_{\alpha] \delta]},
$$

or spelled out

$$
\begin{aligned}
{\left[\mathbf{N}_{a b}, \mathbf{g}_{c d}\right] } & =2 \mathbf{g}_{a[d} \delta_{c] b} \\
{\left[\mathbf{t r}_{a b}, \mathbf{g}_{c d}\right] } & =4 \delta_{[c[a} \mathbf{N}_{d] b]}+2 d \delta_{a[d} \delta_{c] b}, \\
{\left[\mathbf{N}_{a b}, \mathbf{t r}_{c d}\right] } & =-2 \operatorname{tr}_{b[d} \delta_{c] a}, \\
{\left[\mathbf{N}_{a b}, \mathbf{N}_{c d}\right] } & =\delta_{b c} \mathbf{N}_{a d}-\delta_{a d} \mathbf{N}_{c b}
\end{aligned}
$$

Their Casimir (15) is

$$
\mathbf{c}=\mathbf{g}_{a b} \mathbf{t r}_{b a}+\mathbf{N}_{a b} \mathbf{N}_{b a}-(d+q-1) \mathbf{N}_{a a}+\frac{1}{4} d q(d+2 q-2) .
$$

The operators $v_{\alpha}$ generalize the exterior derivative and codifferential

$$
\left(v_{\alpha}\right)=\left(\begin{array}{c}
\mathbf{d}_{1} \\
\vdots \\
\mathbf{d}_{q} \\
\boldsymbol{\delta}_{1} \\
\vdots \\
\boldsymbol{\delta}_{q}
\end{array}\right) .
$$

These act on each antisymmetric set of indices much like the usual $\mathbf{d}$ and $\boldsymbol{\delta}$ operators, but it is important to note that even the operators $\mathbf{d}_{a} \cong d x_{a}^{\mu} D_{\mu}$ 
are not metric independent, since they employ the covariant derivative. The $v_{\alpha}$ transform as a vector under ${ }^{13} s o(2 q)$

$$
\begin{array}{ll}
{\left[\mathbf{N}_{a b}, \mathbf{d}_{c}\right]=\delta_{b c} \mathbf{d}_{a},} & {\left[\mathbf{N}_{a b}, \boldsymbol{\delta}_{c}\right]=-\delta_{a c} \boldsymbol{\delta}_{b},} \\
{\left[\mathbf{t r}_{a b}, \mathbf{d}_{c}\right]=2 \delta_{c[b} \boldsymbol{\delta}_{a]},} & {\left[\mathbf{g}_{a b}, \boldsymbol{\delta}_{c}\right]=2 \delta_{c[b} \mathbf{d}_{a]} .}
\end{array}
$$

They are supercharges from the spinning particle model viewpoint and obey the superalgebra (49). In detail, these read

$$
\begin{aligned}
& \left\{\mathbf{d}_{a}, \mathbf{d}_{b}\right\}=R_{\mu \nu \rho \sigma} d x_{a}^{\mu} d x_{b}^{\nu} d x_{c}^{\rho} \partial_{c}^{\sigma} \\
& \left\{\mathbf{d}_{a}, \boldsymbol{\delta}_{b}\right\}=\delta_{a b} \Delta+R_{\mu \nu \rho \sigma} d x_{a}^{\mu} \partial_{b}^{\nu} d x_{c}^{\rho} \partial_{c}^{\sigma}, \\
& \left\{\boldsymbol{\delta}_{a}, \boldsymbol{\delta}_{b}\right\}=R_{\mu \nu \rho \sigma} \partial_{a}^{\mu} \partial_{b}^{\nu} d x_{c}^{\rho} \partial_{c}^{\sigma} .
\end{aligned}
$$

Let us spell those relations out explicitly for the constant curvature case (79)

$$
\begin{aligned}
& \left\{\mathbf{d}_{a}, \mathbf{d}_{b}\right\}=2 \mathbf{g}_{c(a} \mathbf{N}_{b) c}, \\
& \left\{\mathbf{d}_{a}, \boldsymbol{\delta}_{b}\right\}=\delta_{a b}\left(\square-\mathbf{c}+\frac{1}{4} d q(d+2 q-2)\right) \\
& +\mathbf{g}_{a c} \mathbf{t r}_{c b}+\mathbf{N}_{a c} \mathbf{N}_{c b}-(d+q-1) \mathbf{N}_{a b}, \\
& \left\{\boldsymbol{\delta}_{a}, \boldsymbol{\delta}_{b}\right\}=2 \mathbf{N}_{c(a} \mathbf{t r}_{b) c},
\end{aligned}
$$

where the Laplacian is related to the Lichnerowicz wave operator by

$$
\square=\Delta+\mathbf{c}-\frac{1}{4} q d(d+2 q-2) .
$$

\subsection{Super-Lichnerowicz Algebras}

It is now clear that our supersymmetric quantum mechanical system provides a vast generalization of Lichnerowicz's original construction. The most general extension acts on tensors with $p$ sets of symmetrized indices, $[Q / 2]$ sets of anti-symmetrized indices and a single spinor index present only for $Q$ odd

$$
\left.\Phi_{\left(\mu_{1} \ldots \mu_{s_{1}}\right), \cdots,\left(\mu_{1} \ldots \mu_{s_{p}}\right) ;\left[\nu_{1} \ldots \nu_{k_{1}}\right], \cdots,\left[\nu_{1} \ldots \nu_{k}[Q / 2]\right.}^{\alpha}\right] .
$$

\footnotetext{
${ }^{13}$ We apologize for ambiguous notations such as: (i) the Kronecker delta $\delta_{a b}$ versus codifferentials, $\boldsymbol{\delta}_{a}$, (ii) the spacetime dimension $d=\operatorname{dim}_{M}$ versus exterior derivatives $\mathbf{d}_{a}$, and (iii) the use of $\alpha$ as both an $\operatorname{osp}(2 p \mid Q)$ superindex and as a spacetime spinor index.
} 
In Young diagram notation, where rows are totally symmetric and columns antisymmetric, we could write

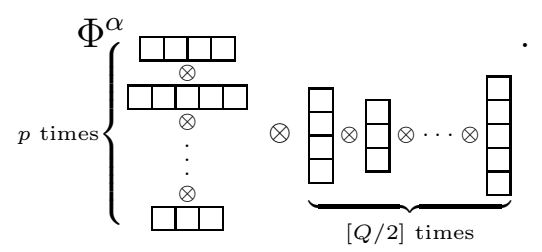

Clearly, although this is not an irreducible basis for tensors and spinors on a manifold, we can generate all such objects this way. Indeed, the study of irreducible tensors amounts to an examination of irreducible representations of the non-compact orthosymplectic algebra obeyed by the conserved charges. A detailed study is reserved for future work, however.

Firstly, let us discuss the $\operatorname{osp}(2 p \mid Q)$ generators. For any $Q$, these can (i) add a pair of boxes, (ii) count or move boxes and (iii) remove a pair of boxes using $\mathbf{g}, \mathbf{N}$ and tr. These are now operator-valued supermatrices. Their fermionic entries act on pairs of boxes of which one lives in an (antisymmetric) column, and the other in a (symmetric) row. Otherwise, they act exactly as in the above multisymmetric tensor and multiform examples. When $Q$ is odd, states are also labeled by a spinor index, so a single box can be added or removed using a Dirac matrix as described in section 4.4. All these operators fit into the conserved charges $f_{\alpha \beta}$ according to

$$
\left(f_{\alpha \beta}\right)=\left(\begin{array}{cc||cc|c}
\mathbf{g}_{i j} & \mathbf{N}_{i j}+\frac{d}{2} \delta_{i j} & \mathbf{g}_{i b} & \mathbf{N}_{i b} & \frac{1}{\sqrt{2}} \boldsymbol{\gamma}_{i} \\
\mathbf{N}_{j i}+\frac{d}{2} \delta_{i j} & \mathbf{t r}_{i j} & \mathbf{N}_{b i} & \mathbf{t r}_{i b} & \frac{1}{\sqrt{2}} \boldsymbol{\gamma}_{i}^{*} \\
\hline \hline \mathbf{g}_{a j} & \mathbf{N}_{a j} & \mathbf{g}_{a b} & \mathbf{N}_{a b}-\frac{d}{2} \delta_{a b} & \frac{1}{\sqrt{2}} \boldsymbol{\gamma}_{a} \\
\mathbf{N}_{j a} & \mathbf{t r}_{a j} & -\mathbf{N}_{b a}+\frac{d}{2} \delta_{a b} & \mathbf{t r}_{a b} & \frac{1}{\sqrt{2}} \boldsymbol{\gamma}_{a}^{*} \\
\hline \frac{1}{\sqrt{2}} \gamma_{j} & \frac{1}{\sqrt{2}} \gamma_{j}^{*} & -\frac{1}{\sqrt{2}} \gamma_{b} & -\frac{1}{\sqrt{2}} \boldsymbol{\gamma}_{b}^{*} & 0
\end{array}\right) .
$$

The final column and row should be omitted for $Q$ even. Here $i, j, \ldots$ and $a, b, \ldots$ take values $1, \ldots, p$ and $1, \ldots,[Q / 2]$. The operator matrix entries are defined by

$$
\mathbf{g}_{\bullet} \equiv d x_{\bullet}^{\mu} g_{\mu \nu} d x_{\circ}^{\nu}, \quad \mathbf{N}_{\bullet} \equiv d x_{\bullet}^{\mu} g_{\mu \nu} \partial_{\circ}^{\nu}, \quad \operatorname{tr}_{\bullet} \equiv \partial_{\bullet}^{\mu} g_{\mu \nu} \partial_{\circ}^{\nu},
$$




$$
\gamma_{\bullet} \equiv d x_{\bullet}^{\mu} \gamma_{\mu}, \quad \gamma_{\bullet}^{*} \equiv \partial_{\bullet}^{\mu} \gamma_{\mu} .
$$

where $\bullet$ and $\circ$ stand for indices of either type $i, j, \ldots$ or $a, b, \ldots$ The differentials and their duals are either commuting or anticommuting with nonvanishing brackets

$$
\left[\partial_{i}^{\mu}, d x_{j}^{\nu}\right]=\delta_{i j} g^{\mu \nu}, \quad\left\{\partial_{a}^{\mu}, d x_{b}^{\nu}\right\}=\delta_{a b} g^{\mu \nu} .
$$

In terms of the oscillators the correspondence is $X_{\alpha}^{\mu}=\left(d x_{i}^{\mu}, \partial_{i}^{\mu}, d x_{a}^{\mu}, \partial_{a}^{\mu}, \frac{1}{\sqrt{2}} \gamma^{\mu}\right)$. The superalgebra of the $\operatorname{ssp}(2 p \mid Q)$ generators $f_{\alpha \beta}$ is given in (49) and their quadratic Casimir by (15).

Our discussion so far holds for any Riemannian manifold. It remains to discuss the charges $v_{\alpha}=i X_{\alpha}^{\mu} \pi_{\mu}$ and the Lichnerowicz wave operator $\square=-2 H$. These operators can distinguish between general backgrounds, symmetric spaces, and constant curvature ones. The operator $i \pi_{\mu}$ corresponds to the covariant derivative operator on states (see equation (40)). For $\alpha=a$ odd, these are "standard" supersymmetry operators. From a geometric viewpoint, this means that they act on columns (i.e., form indices) as exterior derivative and codifferential operators. It is important to realize that the covariant derivative $D_{\mu}$ acting on a tensor-valued differential form $\varphi_{\left[\mu_{1} \ldots \mu_{k}\right] \nu_{1} \ldots \nu_{s}}$ (say) also mixes the tensor indices $\nu_{i}$ through the Christoffel symbols. When $Q$ is odd, the final generator $v_{2 p+Q}$ is the Dirac operator, as discussed in section 4.4. Finally for values of the superindex $\alpha=i$ even, the $v_{\alpha}$ are gradient and divergence operators acting on symmetric rows. In an equation, $v_{\alpha}$ is a column vector

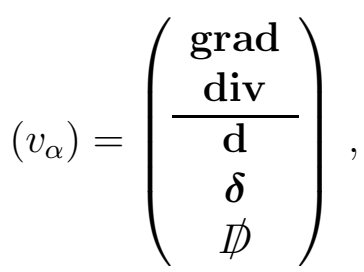

which transforms as the fundamental representation of $\operatorname{osp}(2 p \mid Q)$ as given in (49). Here, omit the final entry from $\left(v_{\alpha}\right)$ when $Q$ is even. The Lichnerowicz wave operator ${ }^{14}$ is again a modification of the Laplacian according to

$$
\square=\Delta+\frac{1}{4} R^{\# \#}+\frac{1}{8} \delta_{2 p+Q, 1} R .
$$

\footnotetext{
${ }^{14}$ It is interesting to note that $\square$ can always be expressed in terms of the $v_{\alpha}$ by con-
} 
It commutes with $f_{\alpha \beta}$ in any background and with $v_{\alpha}$ in symmetric spaces. The algebra of all the above geometric operations is given in (49) and is valid for any Riemannian manifold. We propose to call this a "super-Lichnerowicz algebra". The additional relation (52), required for $\square$ to be central always holds for $(2 p \mid Q)=(0 \mid 1,2)(\mathcal{N}=1,2$ supersymmetry $)$ and is otherwise valid in symmetric spaces $(26)$.

We end this section with its specialization to constant curvature manifolds (61). In that case the operator

$$
R^{\# \#}=4 \mathbf{c}-\frac{1}{2} d(Q-2 p)(Q-2 p+d-2),
$$

i.e., the $\operatorname{osp}(2 p \mid Q)$ quadratic Casimir. The supercommutators of the $v_{\alpha}$ then simplify to

$$
\left[v_{\alpha}, v_{\beta}\right\}=J_{\alpha \beta} \Delta+f_{[\alpha \mid \gamma} J^{\delta \gamma} f_{\delta \mid \beta)}-\frac{1}{4} d(Q-2 p+d-2) J_{\alpha \beta} .
$$

The Lichnerowicz wave operator is simply

$$
\square=\Delta+\mathbf{c}-\frac{1}{8} d(Q-2 p)(Q-2 p+d-2)+\frac{1}{8} \delta_{2 p+Q, 1} R .
$$

\subsection{Parabolic Orthosymplectic Algebra}

The superalgebra (49) is certainly consistent since it was defined by the charges of a quantum mechanical system acting on an explicit representation by quantum states or equivalently tensors on a manifold. However, the presence of terms quartic in the oscillators $X_{\alpha}^{m}$ on the right hand side of the $[v, v\}$-supercommutator imply that this is not a closed algebra. (The reader may also easily convince themselves of this fact by studying the constant curvature, $(2 p, Q)=(2,0)$ case studied in depth in [8].) However, when the curvature vanishes, we do obtain a finite dimensional Lie superalgebra. As we now show, it is a parabolic subalgebra of $\operatorname{osp}(2 p+2 \mid Q)$. The curved models are deformations of this parabolic algebra.

tracting the $\left[v_{\alpha}, v_{\beta}\right\}$ bracket with $J^{\alpha \beta}$. Moreover, when $Q \neq 2 p$ an even simpler formula

$$
\square=\frac{1}{p-Q / 2} v \cdot v+\frac{1}{4} \frac{p-Q / 2+1}{p-Q / 2} R^{\# \#}+\frac{1}{8} \delta_{2 p+Q, 1} R .
$$

holds upon contracting with $J^{\beta \alpha}$. 
The first step is to introduce a new operator ord which counts derivatives:

$$
[\text { ord }, H]=2 H, \quad\left[\text { ord }, v_{\alpha}\right]=v_{\alpha}, \quad\left[\text { ord }, f_{\alpha \beta}\right]=0 .
$$

Although ord is not a conserved charge, it can be interpreted as a dilation generator, and will play an extremely important rôle. In particular, it provides a 3-grading of the Lie superalgebra

$$
\mathfrak{p}=\mathfrak{g}_{2} \oplus \mathfrak{g}_{1} \oplus \mathfrak{g}_{0},
$$

where $\mathfrak{g}_{2}=\{H\}, \mathfrak{g}_{1}=\left\{v_{\alpha}\right\}$ and $\mathfrak{g}_{0}=\left\{f_{\alpha \beta}\right.$, ord $\}$. Moreover, $\left\{H, \mathfrak{g}_{1}\right.$, ord $\}$ form a Heisenberg Lie superalgebra

$$
\left[v_{\alpha}, v_{\beta}\right\}=-2 J_{\alpha \beta} H \text {. }
$$

It is natural to wonder therefore, whether the algebra $\mathfrak{p}$ is the parabolic subalgebra of some larger Lie superalgebra, and indeed this is the case. Namely, the Lie superalgebra $\operatorname{osp}(2 p+2 \mid Q)$ has a five grading by the Cartan generator, which we are here calling ord, corresponding to the longest root of its bosonic $s p(2 p+2)$ subalgebra

$$
\operatorname{osp}(2 p+2 \mid Q)=\mathfrak{g}_{2} \oplus \mathfrak{g}_{1} \oplus \mathfrak{g}_{0} \oplus \mathfrak{g}_{-1} \oplus \mathfrak{g}_{-2} .
$$

The non-negatively graded subspaces are isomorphic to the parabolic algebra $\mathfrak{p}$, while we have as yet found no physical interpretation of the negatively graded subspaces, but cannot help remarking that the corresponding quantum mechanical generators ought be computable in terms of inverse powers of $H$.

In summary, the algebra of conserved charges corresponds to all $o s p(2 p+$ $2 \mid Q)$ generators that commute with the generator $H$ labeled by the longest root of the bosonic $s p(2 p+2)$ subalgebra. To verify this claim explicitly, it suffices to display an upper-triangular matrix representation of the super Lie algebra $\mathfrak{p}$. This is achieved by the $\operatorname{osp}(2 p+2 \mid Q)$ supermatrix

$$
\mathcal{P} \equiv\left(\begin{array}{c|c|c}
\text { ord } & v_{\alpha} & 4 H \\
\hline 0 & 2 J^{\beta \gamma} f_{\gamma \alpha} & -J^{\beta \gamma} v_{\gamma} \\
\hline 0 & 0 & \text {-ord }
\end{array}\right)
$$

(it is not difficult to permute the rows and columns of $\mathcal{P}$ to obtain the usual osp-valued block bosonic/fermionic supermatix form). 


\section{Conclusions}

In this article, we have presented curved space spinning particle models whose spin degrees of freedom are described by both fermionic and bosonic oscillators. These transform as spacetime vectors and under the superalgebra $o s p(2 p \mid Q)$. States of these models describe the most general spinors and tensors on any Riemannian manifold. The conserved charges describe the various geometric operators on tensors and spinors. For manifolds that are symmetric spaces, our models are supersymmetric with $Q$ supercharges corresponding to exterior derivatives, codifferentials, and (when $Q$ is odd) the Dirac operator. Moreover, the underlying superalgebra is a deformation of a parabolic subalgebra of $\operatorname{osp}(2 p+2 \mid Q)$ with $2 p$ "commuting" supercharges which act as gradient and divergence operators. The Hamiltonian of the model is a modified Laplacian that generalizes Lichnerowicz's constant curvature wave operator to tensors and spinors on arbitrary Riemannian manifolds. The remaining charges amount to all possible operations on tensors and spinors using the metric and Dirac matrices.

There are many applications, generalizations, and open directions suggested by our work. We close by giving a (partial) list:

1. In special geometries it is possible to write down quantum mechanical systems with higher supersymmetries [33, 34], the first example being Kähler backgrounds [3]. It is possible motivate the super-Lichnerowicz algebra presented here by analogy with the Hodge-Lefshetz algebra of Dolbeault cohomology which is also described in terms of supersymmetric quantum mechanics [5]. It would be interesting to consider

Kähler backgrounds for our models to try and marry the two algebras (an investigation of this direction may be found in [35]).

2. We have said nothing here about the spectrum of the model or its detailed dynamics. Eigenmodes of Lichnerowicz operators have been studied before $[23,36]$, but it would be interesting to have statements applicable to our more general models. Clearly this is an important problem deserving further attention.

3. An important relation of supersymmetric quantum mechanics to geometry is through index theorems. The Lichnerowicz operators presented here are natural regulators for index computations in an extremely broad class of spaces, in particular, from a geometric standpoint the 
quantum mechanical trace over states runs over infinitely many fields. Whether novel invariants can be constructed this way is an open problem.

4. Second quantization of these models introduces fields of arbitrary spin, thanks to the introduction of bosonic oscillators $[14,15]$. Already the Lichnerowicz algebra for the $\operatorname{osp}(2 \mid 0)$ model implies generating functions for totally massless, symmetric, higher spin field actions. Indeed, calling

$$
\begin{aligned}
G & =\square+2(\mathbf{N}-1)(\mathbf{N}+d-3)-\operatorname{grad} \operatorname{div}+\frac{1}{2}\left(\operatorname{grad}^{2} \operatorname{tr}+\mathbf{g} \mathbf{d i v}^{2}\right) \\
& -\frac{1}{2} \mathbf{g}\left(\square+2 \mathbf{N}(\mathbf{N}+d-1)+d-1+\frac{1}{2} \operatorname{grad} \operatorname{div}\right) \mathbf{t r},
\end{aligned}
$$

the (Bianchi) identity

$$
\operatorname{div} G=\mathbf{g} X=0 \bmod \mathbf{g},
$$

implies that the action

$$
S=\int \Phi^{*} G \Phi=\langle\Phi|G| \Phi\rangle
$$

is gauge invariant under $\delta \Phi=\operatorname{grad} \xi$ when $\operatorname{tr} \xi=0$. This is the invariance of a doubly trace free, $\operatorname{tr}^{2} \Phi=0$, field. Since $\Phi$ need not be an eigenstate of the index operator $\mathbf{N}$, the action (131) generates the actions of all totally symmetric, massless fields in constant curvature backgrounds.

Similarly, the $\operatorname{osp}(2 \mid 1)$ model pertains to totally symmetric tensorspinors. Indeed the operator

$$
\begin{aligned}
G & =\mathcal{D}-2 \mathbf{N} \not D+(d-4)\left[\boldsymbol{\gamma} \not D \boldsymbol{\gamma}^{*}-\mathcal{G} \mathbf{r a d} \boldsymbol{\gamma}^{*}-\boldsymbol{\gamma} \mathcal{D} \mathbf{i v}\right] \\
& +\frac{1}{2}(d-2)\left[\boldsymbol{\gamma} \mathcal{G} \mathbf{r a d} \mathbf{t r}+\mathbf{g} \mathcal{D} \mathbf{i v} \boldsymbol{\gamma}^{*}-i(2 \mathbf{N}+d-4)\right] \\
& -\frac{1}{4}(d-2)\left[\mathbf{g}\left(\not D-\frac{i}{2}(2 \mathbf{N}+d)\right) \mathbf{t r}-2 i \boldsymbol{\gamma}(2 \mathbf{N}+d-2) \boldsymbol{\gamma}^{*}\right]
\end{aligned}
$$


obeys the identity

$$
\mathcal{D i v} G=\gamma X=0 \bmod \gamma
$$

so that the action

$$
S=\int \bar{\Psi} G \Psi=\langle\Psi|G| \Psi\rangle
$$

enjoys the gauge invariance $\delta \Psi=\overline{\mathcal{G} \text { rad }} \xi$ with $\gamma \xi=0$, of a traceless, gamma-traceless, $\gamma^{*} \operatorname{tr} \Psi=0$, fermionic higher spin field.

An obvious approach for obtaining the above actions is to gauge the rigid osp symmetries [9] of the models. Spinning particle studies of this type have been conducted in [29, 30, 13, 37, 38]. Interactions would amount to second quantization of these spinning particle models. Needless to say, this is an ambitious program, see [39] for a review of interacting higher spin theories.

5. The super-Lichnerowicz algebra is a deformation of a parabolic $o s p(2 p+$ $2 \mid Q)$ algebra. It would be most interesting to find geometric operators corresponding to the remaining $\operatorname{osp}(2 p+2 \mid Q)$ operators. In particular, it seems likely that introducing the Green's function $\square^{-1}$ may yield a solution to this problem. The additional charges would not be expected to yield quantum mechanical symmetries, but instead ought be spectrum generating.

6. The Lichnerowicz algebra for the $\operatorname{osp}(2 \mid 0)$ model is a deformation of the Jacobi group $G^{J}$, an object extensively studied in the mathematical literature (see the excellent book [25]). In particular it is known that this group enjoys a cubic Casimir operator, in our notations

$$
\mathbf{c}_{3}=\square\left(\mathbf{c}+\mathbf{N}+\frac{d(d-4)}{4}\right)-\operatorname{grad}^{2} \operatorname{tr}-\mathbf{g} \operatorname{div}^{2}+\operatorname{grad}(2 \mathbf{N}+d-2) \operatorname{div}
$$

This operator is central in flat backgrounds. We know of no generalization to curved backgrounds however.

7. Instead of studying the model via the Lie algebra obeyed by its conserved charges, for the $\operatorname{osp}(2 \mid 0)$ theory it is also helpful to work in an extended universal enveloping algebra. As shown in [8], in this case 
there is a rather elegant associative algebra. The key step is to enlarge the constant curvature algebra by a certain square root of the Casimir

$$
\mathcal{T} \equiv-\sqrt{1-\mathbf{c}}
$$

and in addition we define $\mathcal{N} \equiv \mathbf{N}+\frac{d}{2}-1$. This allows us to form the operator $\mathcal{N}+\mathcal{T}$ whose eigenstates are $k$-fold trace-free tensors, namely

$$
\operatorname{tr}^{k} \varphi=0 \neq \operatorname{tr}^{k-1} \varphi \Longrightarrow(\mathcal{N}+\mathcal{T}) \varphi=2 k \varphi
$$

Then introducing

$$
\widetilde{\operatorname{div}} \equiv(\mathcal{N}-\mathcal{T}) \operatorname{div}-\operatorname{grad} \operatorname{tr}
$$

and similarly for the formal adjoint $\widetilde{\text { grad}}$, the constant curvature algebra is presented by the six relations

$$
\begin{gathered}
\operatorname{tr} \mathcal{N}=(\mathcal{N}+2) \operatorname{tr}, \quad \operatorname{tr} \widetilde{\operatorname{grad}}=\widetilde{\operatorname{grad}} \frac{\mathcal{N}-\mathcal{T}+4}{\mathcal{N}-\mathcal{T}+2} \operatorname{tr}, \\
\operatorname{gtr}=\mathcal{N}^{2}-\mathcal{T}^{2}=\operatorname{tr} \mathrm{g}-4 \mathcal{N}-4, \\
\widetilde{\operatorname{div}} \mathcal{T}=(\mathcal{T}-1) \widetilde{\operatorname{div}}, \quad \widetilde{\operatorname{div}} \mathcal{N}=(\mathcal{N}+1) \widetilde{\operatorname{div}} \\
\widetilde{\operatorname{div}} \widetilde{\operatorname{grad}}=\widetilde{\operatorname{grad}} \widetilde{\operatorname{div}} \frac{(\mathcal{N}-\mathcal{T}+2) \mathcal{T}^{2}}{(\mathcal{N}-\mathcal{T})\left(\mathcal{T}^{2}-1\right)} \\
-2 \frac{\left(\square-\frac{(d-2)^{2}}{2}+2 \mathcal{T}^{2}\right)(\mathcal{N}-\mathcal{T}+2) \mathcal{T}^{2}}{\mathcal{N}-\mathcal{T}}
\end{gathered}
$$

and their formal adjoints where all other products are commutative. In particular, observe that the $s p(2, \mathbb{R})$ action on the pair $(\widetilde{\mathbf{d i v}}, \widetilde{\text { grad }})$ is diagonal. These relations provide a calculus for constant curvature algebra computations in terms of rational functions of $(\mathcal{N}, \mathcal{T})$. Needless to say, it would be extremely fruitful to generalize this associative algebra to the general $\operatorname{osp}(2 p \mid Q)$ models.

\section{Acknowledgments}

It is a pleasure to thank Boris Pioline, Sergey Prokushkin, and Andrew Hodge for discussions. 


\section{References}

[1] L. Alvarez-Gaume and E. Witten, Nucl. Phys. B 234, 269 (1984).

[2] E. Witten, J. Diff. Geom. 17, 661 (1982).

[3] B. Zumino, Phys. Lett. B 87, 203 (1979).

[4] E. Witten, Nucl. Phys. B 202, 253 (1982).

[5] J. M. Figueroa-O'Farrill, C. Kohl and B. J. Spence, Nucl. Phys. B 503, 614 (1997) [arXiv:hep-th/9705161].

[6] A. Lichnerowicz, Institut des Hautes Études Scientifiques, 10, 293 (1961); Bull. Soc. Math. France, 92, 11 (1964).

[7] C. Duval, P. Lecomte, V. Ovsienko, Ann. Inst. Fourier, 49, 1999 (1999); C. Duval and V. Ovsienko, Selecta Math. (N.S.), 7, 291 (2001).

[8] K. Hallowell and A. Waldron, Nucl. Phys. B 724453 (2005) [arXiv:hepth/0505255].

[9] L. Brink, S. Deser, B. Zumino, P. Di Vecchia and P. S. Howe, Phys. Lett. B 64, 435 (1976).

[10] R. H. Rietdijk and J. W. van Holten, Class. Quant. Grav. 7, 247 (1990).

[11] G. W. Gibbons, R. H. Rietdijk and J. W. van Holten, Nucl. Phys. B 404, 42 (1993) [arXiv:hep-th/9303112].

[12] V. D. Gershun and V. I. Tkach, Pisma Zh. Eksp. Teor. Fiz. 29320 (1979) [Sov. Phys. JETP 29288 (1979)]. P. S. Howe, S. Penati, M. Pernici and P. K. Townsend, Phys. Lett. B 215555 (1988); Class. Quant. Grav. 6 1125 (1989).

[13] F. Bastianelli, O. Corradini and E. Latini, "Higher spin fields from a worldline perspective", [arXiv:hep-th/0701055].

[14] J. M. F. Labastida, Nucl. Phys. B 322, 185 (1989).

[15] M. A. Vasiliev, Phys. Lett. B 209, 491 (1988). 
[16] M. A. Vasiliev, Phys. Lett. B 243, 378 (1990); Phys. Lett. B 567, 139 (2003). See also A. Sagnotti, E. Sezgin and P. Sundell, "On higher spins with a strong $\operatorname{Sp}(2, \mathbb{R})$ condition," [arXiv:hep-th/0501156].

[17] S. Deser and A. Waldron, Phys. Rev. Lett. 87, 031601 (2001) [arXiv:hepth/0102166]; Nucl. Phys. B 607, 577 (2001) [arXiv:hep-th/0103198].

[18] R. R. Metsaev, Phys. Lett. B 643, 205 (2006) [arXiv:hep-th/0609029].

[19] P. de Medeiros and C. Hull, JHEP 0305, 019 (2003) [arXiv:hepth/0303036]; Commun. Math. Phys. 235, 255 (2003) [arXiv:hepth/0208155].

[20] M. Dubois-Violette and M. Henneaux, Lett. Math. Phys. 49, 245 (1999) [arXiv:math.qa/9907135]; S. B. Edgara and J. M. M. Senovilla, J. Geom. Phys., 56, 2153(2006); P. J. Olver, "Differential hyperforms I," Univ. of Minnesota report 82-101; "Invariant theory and differential equations," in S. Koh, Invariant theory (Springer-Verlag, 1987) 62; M. Dubois-Violette and M. Henneaux, Lett. Math. Phys. 49 (1999) 245 [math.qa/9907135]; Commun. Math. Phys. 226 (2002) 393 [ math.qa/0110088].

[21] X. Bekaert and N. Boulanger, "Tensor gauge fields in arbitrary representations of GL(D,R). II: Quadratic actions," [arXiv:hep-th/0606198]; Phys. Lett. B 561 (2003) 183 [arXiv:hep-th/0301243].

[22] A. A. Deriglazov and D. M. Gitman, Mod. Phys. Lett. A 14, 709 (1999) [arXiv:hep-th/9811229].

[23] S. M. Christensen and M. J. Duff, Nucl. Phys. B 154, 301 (1979).

[24] N. P. Warner, Proc. Roy. Soc. Lond. A 383, 217 (1982).

[25] R. Berndt and R. Schmidt, "Elements of the representation theory of the Jacobi group", Rolf Berndt, Ralf Schmidt. Birkhäuser Verlag, Boston1998.

[26] S. Deser and B. Zumino, Phys. Rev. Lett. 38, 1433 (1977).

[27] P. K. Townsend, Phys. Rev. D 15, 2802 (1977). 
[28] S. M. Kuzenko and Z. V. Yarevskaya, Mod. Phys. Lett. A 11, 1653 (1996) [arXiv:hep-th/9512115].

[29] P. S. Howe, S. Penati, M. Pernici and P. K. Townsend, Phys. Lett. B 215, 555 (1988).

[30] P. S. Howe, S. Penati, M. Pernici and P. K. Townsend, Class. Quant. Grav. 6, 1125 (1989).

[31] F. Bastianelli, P. Benincasa and S. Giombi, JHEP 0504, 010 (2005) [arXiv:hep-th/0503155].

[32] F. Bastianelli, P. Benincasa and S. Giombi, JHEP 0510, 114 (2005) [arXiv:hep-th/0510010].

[33] R. A. Coles and G. Papadopoulos, Class. Quant. Grav. 7, 427 (1990).

[34] C. M. Hull, [arXiv:hep-th/9910028].

[35] N. Marcus, Nucl. Phys. B 439, 583 (1995) [arXiv:hep-th/9409175].

[36] N. P. Warner, Proc. Roy. Soc. Lond. A 383, 207 (1982).

[37] C. M. Hull and J. L. Vazquez-Bello, Nucl. Phys. B 416, 173 (1994) [arXiv:hep-th/9308022].

[38] R. Halling and A. Lindeberg, Class. Quant. Grav. 7, 2341 (1990).

[39] X. Bekaert, S. Cnockaert, C. Iazeolla and M. A. Vasiliev, [arXiv:hepth/0503128]. 